\title{
Topology of the adiabatic potential energy surfaces for the resonance states of the water anion
}

\author{
D. J. Haxton, ${ }^{1,2, *}$ T. N. Rescigno, ${ }^{2, \dagger}$ and C. W. McCurdy ${ }^{3,4,1, \ddagger}$ \\ ${ }^{1}$ Department of Chemistry, University of California, Berkeley, California 94720 \\ ${ }^{2}$ Lawrence Berkeley National Laboratory, \\ Computing Sciences, Berkeley, California 94720 \\ ${ }^{3}$ Departments of Applied Science and Chemistry, \\ University of California, Davis, California 95616 \\ ${ }^{4}$ Lawrence Berkeley National Laboratory, \\ Chemical Sciences, Berkeley, California 94720
}

\begin{abstract}
The potential energy surfaces corresponding to the long-lived fixed-nuclei electron scatering resonances of $\mathrm{H}_{2} \mathrm{O}$ relevant to the dissociative electron attachment process are examined using a combination of $a b$ initio scattering and bound-state calculations. These surfaces have a rich topology, characterized by three main features: a conical intersection between the ${ }^{2} A_{1}$ and ${ }^{2} B_{2}$ Feshbach resonance states; charge-transfer behavior in the $\mathrm{OH}\left({ }^{2} \Pi\right)+\mathrm{H}^{-}$asymptote of the ${ }^{2} B_{1}$ and ${ }^{2} A_{1}$ resonances; and an inherent double-valuedness of the surface for the ${ }^{2} B_{2}$ state the $C_{2 v}$ geometry, arising from a branch-point degeneracy with a ${ }^{2} B_{2}$ shape resonance. In total, eight individual seams of degeneracy among these resonances are located.
\end{abstract}

\footnotetext{
*djhaxton@lbl.gov

$\dagger$ tnrescigno@lbl.gov

${ }^{\ddagger}$ cwmc curdy@lbl.gov
} 


\section{INTRODUCTION}

We recently reported calculations of the cross sections for dissociative attachment through the lowest-energy resonance [1,2] in electron-water collisions that incorporated a full quantum treatment of the nuclear motion of the resonant state. That study found good agreement with experiment for dissociative attachment through the lowest resonance state $\left({ }^{2} B_{1}\right)$ of the water anion to produce $\mathrm{H}^{-}$, and it established that the associated dynamics are intrinsically polyatomic and thus cannot be described successfully by one-dimensional models.

Dissociative attachment to water is known to also proceed through two other metastable electronic states of the $\mathrm{H}_{2} \mathrm{O}^{-}$anion. At the equilibrium geometry of the water molecule the three states of the water anion have vertical transition energies of approximately $6.5\left({ }^{2} B_{1}\right)$, $8.5\left({ }^{2} A_{1}\right)$, and $11.5 \mathrm{eV}\left({ }^{2} B_{2}\right)$. Our purpose here is to describe the larger picture that has emerged from the combination of our previous calculations of the potential energy surface for the ${ }^{2} B_{1}$ state with preliminary calculations of the potential energy surfaces of the ${ }^{2} A_{1}$ and ${ }^{2} B_{2}$ resonance states.

We have found that these potential surfaces have a number of intersections, at least one of which - namely, a conical intersection between the ${ }^{2} A_{1}$ and ${ }^{2} B_{2}$ resonance states - may have a major effect on the dynamics of dissociative attachment to water. Indeed, the calculations we will present here strongly suggest that production of $\mathrm{O}^{-}+\mathrm{H}_{2}$ from dissociative attachment to the ${ }^{2} B_{2}$ state must result from nonadiabatic coupling to the ${ }^{2} A_{1}$ state, since that channel is not an asymptote of the ${ }^{2} B_{2}\left(2^{2} A^{\prime}\right)$ surface.

Polyatomic anions can also pose interesting questions regarding the topology of the dissociation limits of their potential energy surfaces, and those concern us here as well. For example, for a potential surface of $\mathrm{H}_{2} \mathrm{O}^{-}$to dissociate both to $\mathrm{H}_{2}+\mathrm{O}^{-}$in one arrangement and to $\mathrm{H}^{-}+\mathrm{OH}$ in another might not initially appear to raise any topological question. However, dissociating the $\mathrm{H}_{2}$ molecule in one of those limits and dissociating the $\mathrm{OH}$ fragment in the other brings the system to exactly the same geometry of three separated atoms, but with the extra electron on $\mathrm{O}^{-}$in first case and $\mathrm{H}^{-}$in the other - a clear impossibility on a single potential surface.

Metastable states have finite lifetimes, and the associated widths form the imaginary parts of their energies. Therefore the overall topology of this system of potential energy surfaces for the water anion involves intersections of complex-valued surfaces. As we will demonstrate, 
this situation allows topological complications such as branch-point degeneracies between complex potential energy surfaces. We endeavor here to provide a comprehensive view of the topology of the potential energy surfaces of the three resonance states of the water anion that feature in experimental observations of dissociative attachment, and to explore the potential role of their intersections in the dynamics of the dissociative attachment process.

Dissociative attachment of electrons to water molecules was examined experimentally as early as 1930 [3]. Subsequent experiments [4-12] and calculations [10, 13-19] in the decades that followed improved the understanding of this physical process and characterized the three resonances that are responsible for clearly resolved peaks in the cross sections for producing different fragments.

To understand the identities of the three resonances responsible for dissociative attachment in water it useful to recall that the ground state configuration of water is $1 a_{1}^{2}$ $2 a_{1}^{2} 1 b_{2}^{2} 3 a_{1}^{2} 1 b_{1}^{2}$ in its equilibrium geometry, with bond distances equal to $1.81 a_{0}$ (where $a_{0}=0.528 \times 10^{-10} \mathrm{~m}$ is the Bohr radius) and a bond angle of $104.5^{\circ}$. The three resonances are "Feshbach resonances" because they are associated with electronically excited states of the molecule. They are characterized by configurations in which an electron is attached in a $4 a_{1}$ virtual orbital to the state arising from the excitation of an electron from the occupied $1 b_{1}, 3 a_{1}$ or $1 b_{2}$ to the same $4 a_{1}$ virtual orbital. In $\mathrm{C}_{2 v}$ geometries those resonances have, respectively, ${ }^{2} B_{1},{ }^{2} A_{1}$ and ${ }^{2} B_{2}$ symmetry. When the $\mathrm{OH}$ distances are unequal, the molecule belongs to the $\mathrm{C}_{s}$ point group, and the appropriate symmetry labels are ${ }^{2} A^{\prime \prime}\left({ }^{2} B_{1}\right), 1^{2} A^{\prime}$ $\left({ }^{2} A_{1}\right)$, and $2^{2} A^{\prime}\left({ }^{2} B_{2}\right)$. We will see later that the $1^{2} A^{\prime}$ and $2^{2} A^{\prime}$ states undergo a conical intersection with each other, so that strictly speaking they each may be associated with either the ${ }^{2} A_{1}$ or ${ }^{2} B_{2}$ states at various geometries.

These states have a finite lifetime because they are embedded in the electronic continuum of $\mathrm{e}^{-}+\mathrm{H}_{2} \mathrm{O}$. At a fixed nuclear geometry they correspond to the energies of electron scattering resonances with complex-valued energies, $E_{R}-i \Gamma / 2$. Our calculations of the nuclear dynamics of dissociative attachment [1] to this system have employed the Local Complex Potential (LCP) [20-24] model, which uses complex-valued potential energy surfaces just as the Born-Oppenheimer treatment of bound-state dynamics uses real-valued ones. A principal conclusion of the present study is that a complete treatment including all three of the resonance states must involve the dynamics on at least three complex potential surfaces coupled by the appropriate nonadiabatic couplings. 
The outline of this paper is as follows. In Section II we briefly summarize the pertinent experimental results on dissociative attachment and the inverse process of associative detachment. In Section III we describe the features of the ${ }^{2} B_{1}\left({ }^{2} A^{\prime \prime}\right)$ resonance potential energy surface - in particular, its proper asymptotes. In Section IV, we describe the manifold of resonant states of ${ }^{2} A^{\prime}\left({ }^{2} A_{1}\right.$ and $\left.\left.{ }^{2} B_{2}\right)\right)$ symmetry relevant to the dissociative attachment process. We will discuss the fact that this manifold includes not only the ${ }^{2} A_{1}$ and ${ }^{2} B_{2}$ Feshbach resonances, but also a shape resonance of ${ }^{2} B_{2}$ symmetry. We catalog the asymptotes of the Feshbach resonances, and the two interesting features of this manifold: a conical intersection between the ${ }^{2} A_{1}$ and ${ }^{2} B_{2}$ states, and a seam of degeneracy between the ${ }^{2} B_{2}$ shape and Feshbach resonances. We conclude in Section $\mathrm{V}$ with some speculations about the implications of the topology of these potential surfaces for the interpretation of the experimental results on dissociative attachment to water.

\section{THE OBSERVED PRODUCTS OF DISSOCIATIVE ATTACHMENT}

The experimental evidence for the products formed by electron attachment to each of the three resonances yields information about the asymptotes of each of their potential surfaces but also raises questions about their topology. Observations of dissociative attachment to gas-phase water and $\mathrm{D}_{2} \mathrm{O}$ show three peaks in the cross section, corresponding to the three resonant states, near $6.5,8.5$, and $11.5 \mathrm{eV}$. The gross features of the experimental results are that the product arrangement $\mathrm{H}^{-}+\mathrm{OH}\left({ }^{2} \Pi\right)$ is produced from the first two resonance states, and that $\mathrm{H}_{2}+\mathrm{O}^{-}$is produced from all three. In addition, the experiments of Curtis and Walker [12] on $\mathrm{D}_{2} \mathrm{O}$ demonstrated that excited OD is produced from the third peak in the arrangement, $\mathrm{D}^{-}+\mathrm{OD}\left({ }^{2} \Sigma\right)$, along with some ground state products, $\mathrm{D}^{-}+\mathrm{OD}$ $\left({ }^{2} \Pi\right)$, in the same arrangement. These experiments also gave evidence for the opening of the three-body dissociation channel $\mathrm{D}_{2} \mathrm{O}^{-}\left({ }^{2} A_{1}\right) \longrightarrow \mathrm{O}^{-}+\mathrm{D}+\mathrm{D}$ at $8.28 \mathrm{eV}$.

These observations lead us to expect that the two-body asymptotes of the resonant states involved in dissociative attachment to water will include both $\mathrm{OH}\left({ }^{2} \Pi\right)+\mathrm{H}^{-}$, and $\mathrm{OH}\left({ }^{2} \Sigma\right)$ $+\mathrm{H}^{-}$in that arrangement and $\mathrm{H}_{2}+\mathrm{O}^{-}$in the other. We expect that the three-body asymptote $\mathrm{O}^{-}+\mathrm{H}+\mathrm{H}$ will be present on the ${ }^{2} A_{1}$ surface. It is important to note that while $\mathrm{OH}^{-}+\mathrm{H}$ production has been observed in some experiments, it is believed to be a result of dissociative attachment to $\mathrm{H}_{2} \mathrm{O}$ clusters $\left(\mathrm{H}_{2} \mathrm{O}\right)_{n}$ [25]. 
Experiments on the inverse process of associative detachment [26-31] are also pertinent to the question of the topology of the potential surfaces for the three resonances we treat here, and in particular have implications concerning their asymptotes. These experiments demonstrate a large rate of autodetachment for the processes $\mathrm{OH}^{-}+\mathrm{H} \longrightarrow \mathrm{H}_{2} \mathrm{O}+\mathrm{e}^{-}$and $\mathrm{O}^{-}$ $+\mathrm{H}_{2} \longrightarrow \mathrm{H}_{2} \mathrm{O}+\mathrm{e}^{-}$at thermal collision energies, and moreover, that the electron detaches quickly once the anion becomes unbound. These findings indicate that these fragments may correlate to a resonant state of $\mathrm{H}_{2} \mathrm{O}^{-}$which has a large width and an attractive potential energy surface, and is therefore probably not one of the Feshbach resonances suggested by the peaks seen in dissociative attachment and with which our study here begins.

The oxygen anion, $\mathrm{O}^{-}$, has ${ }^{2} \mathrm{P}$ symmetry, and the ground state of $\mathrm{H}_{2}$ has ${ }^{1} \Sigma_{g}$ symmetry, so there are three spatial components of the electronic state of separated $\mathrm{O}^{-}+\mathrm{H}_{2}$. The existence of a very broad shape resonance state in associative detachment that correlates to one of the three spatial components of $\mathrm{O}^{-}+\mathrm{H}_{2}$ would suggest that only the two remaining components can correlate with the Feshbach resonances. Since there are three Feshbach resonances, this means that the asymptotes of those three Feshbach resonance potential surfaces must include a channel besides $\mathrm{O}^{-}+\mathrm{H}_{2}$.

\section{THE ${ }^{2} B_{1}\left({ }^{2} A^{\prime \prime}\right)$ STATE}

\section{A. Two-body asymptotes}

Our previous configuration interaction study of the potential energy surface of the ${ }^{2} B_{1}$ $\left({ }^{2} A^{\prime \prime}\right)$ state [1] indicated that $\mathrm{H}^{-}+\mathrm{OH}\left({ }^{2} \Pi\right)$ and $\mathrm{H}_{2}+\mathrm{O}^{-}$are the correct asymptotes for this state. This potential energy surface and the subsequent study of the quantum nuclear dynamics on the ${ }^{2} B_{1}$ surface [2] were consistent with experimental observations of the total cross section and the degree of vibrational excitation of the $\mathrm{OH}\left({ }^{2} \Pi\right)$ fragment. Also, the production of $\mathrm{O}^{-}+\mathrm{H}_{2}$, though that channel is present as an asymptote of the surface, was very small in comparison to the $\mathrm{H}^{-}+\mathrm{OH}$ channel due to the shape of the potential energy surface. That result is consistent with the experimental observation that much less $\mathrm{O}^{-}$is produced through this resonance than $\mathrm{H}^{-}$. Since the ${ }^{2} B_{1}$ state is the lowest Feshbach resonance and does not appear to interact with any other state, except for the ${ }^{2} A_{1}$ state at linear $\mathrm{H}-\mathrm{O}-\mathrm{H}$ geometry, we are driven to conclude that $\mathrm{H}^{-}+\mathrm{OH}$ and $\mathrm{H}_{2}+\mathrm{O}^{-}$are 
the correct asymptotes for this state, i.e., the observed products from the ${ }^{2} B_{1}$ resonance correspond to different asymptotes of the same adiabatic electronic eigenstate. However, that conclusion raises another question.

\section{B. Three-body breakup paradox}

Given that the ${ }^{2} B_{1}\left({ }^{2} A^{\prime \prime}\right)$ state correlates with $\mathrm{H}_{2}+\mathrm{O}^{-}$in one asymptote and $\mathrm{H}^{-}+\mathrm{OH}$ $\left({ }^{2} \Pi\right)$ in the other, there is an apparent paradox. OH $\left({ }^{2} \Pi\right)$ correlates with $\mathrm{O}\left({ }^{3} P\right)+\mathrm{H}$, and $\mathrm{H}_{2}$ correlates with $\mathrm{H}+\mathrm{H}$. Thus the two paths

1) $\mathrm{H}_{2} \mathrm{O}^{-}\left({ }^{2} B_{1}\right) \longrightarrow \mathrm{H}^{-}+\mathrm{OH} \longrightarrow \mathrm{H}^{-}+\mathrm{H}+\mathrm{O}\left({ }^{3} P\right)$

2) $\mathrm{H}_{2} \mathrm{O}^{-}\left({ }^{2} B_{1}\right) \longrightarrow \mathrm{H}_{2}+\mathrm{O}^{-} \longrightarrow \mathrm{H}+\mathrm{H}+\mathrm{O}^{-}$,

illustrated in Fig. 1, lead to precisely the same geometry but different final states. Which is the correct three-body breakup channel?

The fact that these paths have taken the system adiabatically from one eigenstate at the initial geometry to two different eigenstates at the same final geometry means that a conical intersection of adiabatic surfaces must be crossed by the closed loop that they form. We have performed configuration-interaction (CI) calculations to investigate this question, the details of which calculations are described in Appendix A. Those calculations make use of full CI in an atomic orbital basis that yields the correct energetic ordering of the states of the neutral and anion fragments and thus enables us to follow the resonance surfaces to their asymptotes. From those calculations we have found that the conical intersection that resolves the paradox of the two different three-body breakup states lies effectively at an infinite separation of the atomic and diatomic fragments, and corresponds to a chargetransfer reaction between the fragments.

While the notion of a conical intersection at infinite separation of the fragments may seem strange, it means only that the exact point of degeneracy of the two states is reached only when the fragments do not interact. This behavior corresponds to a charge-transfer reaction that may be understood by referring to the dashed lines in Fig. 2. This figure shows the energies at infinite separation of product states with doublet spin symmetry for either charge arrangement, $\mathrm{OH}^{-}+\mathrm{H}$ and $\mathrm{OH}+\mathrm{H}^{-}$. The potential energy of the adiabatic product state $\mathrm{OH}\left({ }^{2} \Pi\right)+\mathrm{H}^{-}$(for infinite separation between the anion and the diatom) as a function of $\mathrm{OH}$ bond length crosses several states of the opposite charge arrangement. 


\section{$\mathrm{H}_{2} \mathrm{O}^{-}{ }^{2} \mathrm{~B}_{1} \quad \mathrm{OH}(\mathrm{X})+\mathrm{H}^{-}$}

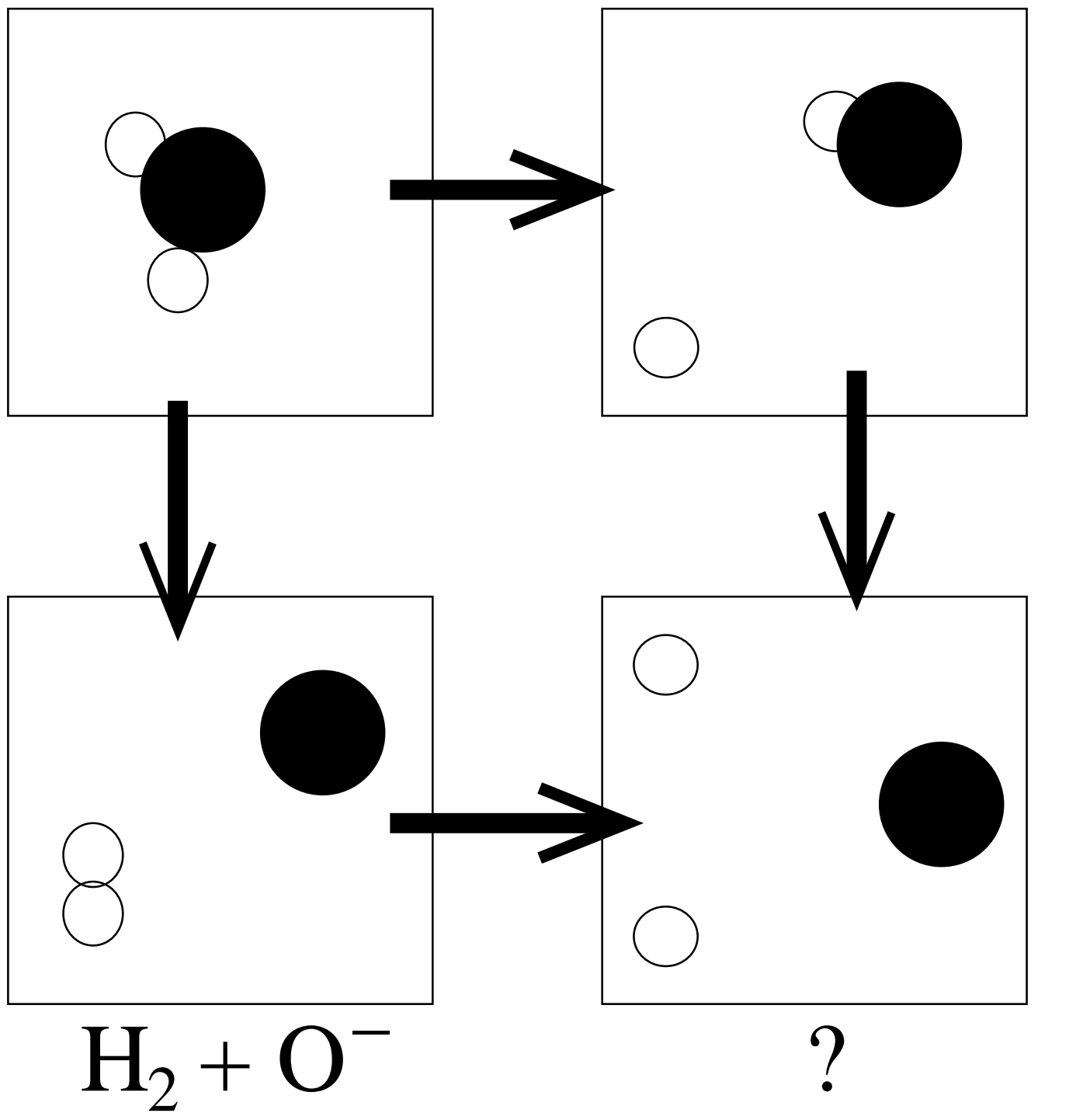

FIG. 1: The ${ }^{2} B_{1}$ state may be taken from the equilibrium geometry of the neutral to the three-body breakup region via either two-body breakup channel, raising the question of which is the proper three-body asymptote.

There are six states of $\mathrm{OH}^{-}$which correlate to $\mathrm{O}^{-}\left({ }^{2} \mathrm{P}\right)+\mathrm{H}$, due to the double spin and triple spatial degeneracy of that product state. The first of these correlates to $\mathrm{OH}^{-}\left(X^{1} \Sigma\right)$ $+\mathrm{H}$ and is not involved here. The second and third of these, which are degenerate, are $\mathrm{OH}^{-}$ $\left({ }^{3} \Pi\right)+\mathrm{H}$ and are the first to intersect the $\mathrm{OH}+\mathrm{H}^{-}$curve as $\mathrm{OH}$ dissociates. Note that the $\mathrm{OH}^{-}\left({ }^{3} \Sigma\right)$ state is unbound at small O-H separation (inward of its crossing with the neutral 


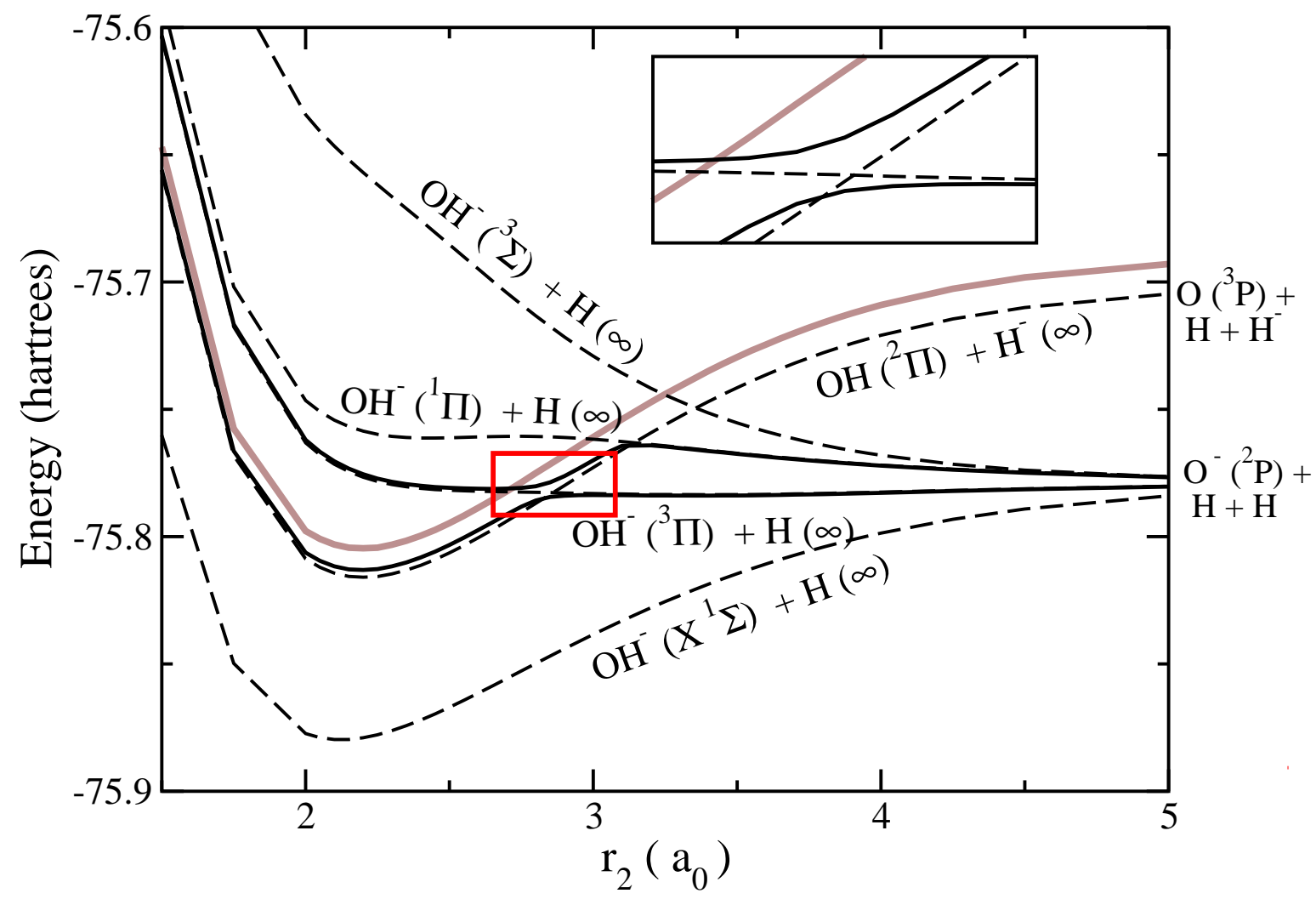

FIG. 2: (Color online) Energies of states involved in charge transfer behavior in $\mathrm{OH}\left({ }^{2} \Pi\right)+\mathrm{H}^{-}$ asymptote of ${ }^{2} B_{1}\left({ }^{2} A^{\prime \prime}\right)$ state, as computed by full CI calculations in an atomic orbital basis, as a function of $\mathrm{O}-\mathrm{H}$ bond length $\mathrm{r}_{2}$. States of $\mathrm{OH}^{-}+\mathrm{H}$, and of $\mathrm{OH}+\mathrm{H}^{-}$, at infinite separation are marked with dashed lines and labeled on the figure. Energies at finite separation of $\mathrm{r}_{1}=10 a_{0}$ $\left(\theta=104.5^{\circ}\right)$ are labeled as follows: anion states, solid black lines; ground state neutral $\mathrm{H}_{2} \mathrm{O}$, wide grey line.

$\mathrm{OH}$ ground state curve), but bound where it crosses $\mathrm{OH}\left({ }^{2} \Sigma\right)+\mathrm{H}^{-}$. The $\mathrm{OH}^{-}\left({ }^{3} \Pi\right)$ state is a shape-resonance type state with configuration $\left[\mathrm{OH}\left({ }^{2} \Pi\right)\right] 4 \sigma^{1}$, that is obtained from the ground state configuration of $\mathrm{OH}^{-}$by promotion of a $\pi$ electron into the antibonding $4 \sigma$ orbital.

As the atomic and diatomic fragments are brought closer and interact, the crossings in Fig. 2 become avoided. In particular, this behavior occurs in the crossing of the $\mathrm{H}^{-}$ $+\mathrm{OH}\left({ }^{2} \Pi\right)$ and $\mathrm{H}+\mathrm{OH}^{-}\left({ }^{3} \Pi\right)$, which is the physically relevant one for understanding the asymptotes of the ${ }^{2} B_{1}\left({ }^{2} A^{\prime \prime}\right)$ surface. In Fig. 2, where results of CI calculations on the anion system at a separation of $10 a_{0}$ are also plotted, it can be seen that the crossing of 
states of opposite charge arrangement at $R_{H-O H}=\infty$ becomes an avoided crossing at finite atom-diatom separation. At this avoided crossing, the ${ }^{2} B_{1}$ resonance switches from being predominantly $\mathrm{H}^{-}+\mathrm{OH}\left({ }^{2} \Pi\right)$ and changes character to the $\mathrm{OH}^{-}\left({ }^{3} \Pi\right)+\mathrm{H}$ state. In other words, the negative charge is moved from the $\mathrm{H}$ to the $\mathrm{OH}$ fragment as the system is brought adiabatically towards three-body breakup. (Similar considerations apply to the crossing of the $\mathrm{H}^{-}+\mathrm{OH}\left({ }^{2} \Pi\right)$ and $\mathrm{H}+\mathrm{OH}^{-}\left({ }^{1} \Pi\right)$ curves, which occurs at larger $\mathrm{OH}$ distances.)

This is a charge-transfer reaction situation but it is geometrically different from the analogous type of crossing in the ion-atom case. In the ion-atom case we see the avoided crossing appear as a function of the interatomic distance, i.e., as a function of the distance across which the charge is being transferred. In this case the avoided crossing occurs at a fixed distance between the fragments as a function of the internal degree of freedom of the diatomic fragment. The geometry at which the intersection occurs $\left(R_{O H} \approx 2.8 a_{0}\right.$ in the calculations in Fig. 2) corresponds to a conical intersection "at infinity" because the $\mathrm{H}^{-}$ fragment is at infinite separation from the diatom. Of course such a conical intersection cannot be circled and so no Berry phase effects could be observed from it.

At finite $\mathrm{H}-\mathrm{OH}$ separations, the accessibility, and probably also the width, of the avoided crossing is no doubt exaggerated in these calculations, because they do not yield an accurate bond energy for the $\mathrm{OH}$. Nonetheless, they suggest the prospect of nonadiabatic coupling to the $\mathrm{OH}^{-}\left({ }^{3} \Pi\right)+\mathrm{H}$ state from high vibrational states of $\mathrm{OH}\left({ }^{2} \Pi\right)+\mathrm{H}^{-}$produced as the ${ }^{2} B_{1}\left({ }^{2} A^{\prime \prime}\right)$ resonance dissociates. Such nonadiabatic coupling would lead to a to selective autodetachment from high $\mathrm{OH}\left({ }^{2} \Pi\right)$ vibrational states. We should note that our previous study $[1,2]$, which did not account for this effect, did in fact predict higher dissociative attachment cross-sections for production of vibrationally excited $\mathrm{OH}$ than has been observed in experiment.

In summary, we understand that the ${ }^{2} B_{1}\left({ }^{2} A^{\prime \prime}\right)$ state correlates with the three-body channel $\mathrm{O}^{-}+\mathrm{H}+\mathrm{H}$, and not $\mathrm{O}+\mathrm{H}^{-}+\mathrm{H}$, because it has an avoided crossing with another bound state as the $\mathrm{OH}$ bond is stretched and the electron jumps from $\mathrm{H}^{-}$to $\mathrm{OH}$.

\section{IV. ${ }^{2} A^{\prime}$ MANIFOLD OF STATES}

We now turn to the description of the numerous interesting features of the ${ }^{2} A^{\prime}$ manifold of resonant states relevant to the dissociative attachment process. This manifold includes 
not only the ${ }^{2} A_{1}$ and ${ }^{2} B_{2}$ Feshbach resonances, but also, as we will demonstrate, a shape resonance of ${ }^{2} B_{2}$ symmetry.

We find two main features of this manifold. First, there is a conical intersection between the ${ }^{2} A_{1}$ and ${ }^{2} B_{2}$ states, which certainly plays a part in the nuclear dynamics associated with dissociative attachment via the ${ }^{2} B_{2}$ Feshbach resonance. Second, the ${ }^{2} B_{2}$ Feshbach resonance interacts with the ${ }^{2} B_{2}$ shape resonance in such a way that these two states are actually two components of a double-valued resonant surface in $\mathrm{C}_{2 v}$ geometries. There is a branch-point degeneracy between these two states which is responsible for this doublevaluedness.

The combination of the conical intersection and the ${ }^{2} B_{2}$ seam of degeneracy will lead to a complicated global topology, once $\mathrm{C}_{2 v}$ symmetry is broken and these three resonances become members of the same $A^{\prime}$ irreducible representation. For this reason, it is difficult to provide a complete representation of the topology of the three members of the ${ }^{2} A^{\prime}$ manifold of states. Therefore we will restrict our discussion here to an analysis of the conical intersection between the Feshbach resonances, the determination of the two-body breakup channels of the Feshbach resonances and a description of the single-valued ${ }^{2} A_{1}$ and double-valued ${ }^{2} B_{2}$ surfaces in $\mathrm{C}_{2 v}$ geometries.

\section{A. Conical intersection of ${ }^{2} A_{1}$ and ${ }^{2} B_{2}$ states}

The dominant configurations of the ${ }^{2} B_{1},{ }^{2} A_{1}$ and ${ }^{2} B_{2}$ states at the equilibrium geometry of the neutral are $\left[\mathrm{H}_{2} \mathrm{O}\right] 1 b_{1}^{-1} 4 a_{1}^{2},\left[\mathrm{H}_{2} \mathrm{O}\right] 3 a_{1}^{-1} 4 a_{1}^{2}$, and $\left[\mathrm{H}_{2} \mathrm{O}\right] 1 b_{2}^{-1} 4 a_{1}^{2}$, respectively. The $1 b_{1}$ orbital is primarily nonbonding in character, being comprised mostly of the oxygen $2 p_{x}$ orbital. (We define the $\mathrm{z}$ axis as the $\mathrm{C}_{2 v}$ axis, and the $\mathrm{x}$ axis as perpendicular to the molecular plane.) The $1 b_{2}$ orbital has a node along the xz plane and is bonding between the oxygen and each hydrogen, and antibonding between the hydrogens. The $3 a_{1}$ orbital has bonding character among all the atoms. Finally, the $4 a_{1}$ orbital is antibonding along the $\mathrm{O}-\mathrm{H}$ bonds and bonding between the hydrogens.

A Walsh diagram, shown in Fig. 3, of SCF orbital energies of ground-state neutral water is useful in understanding the behavior of the resonance potential surfaces. Fixing $r_{1}$ and $r_{2}$ at $1.81 a_{0}$, the energy of the $1 b_{2}$ orbital increases as the $\mathrm{H}-\mathrm{O}-\mathrm{H}$ bond angle is decreased, owing to its antibonding $\mathrm{H}-\mathrm{H}$ character, and the energy of the $3 a_{1}$ orbital decreases. Therefore, 


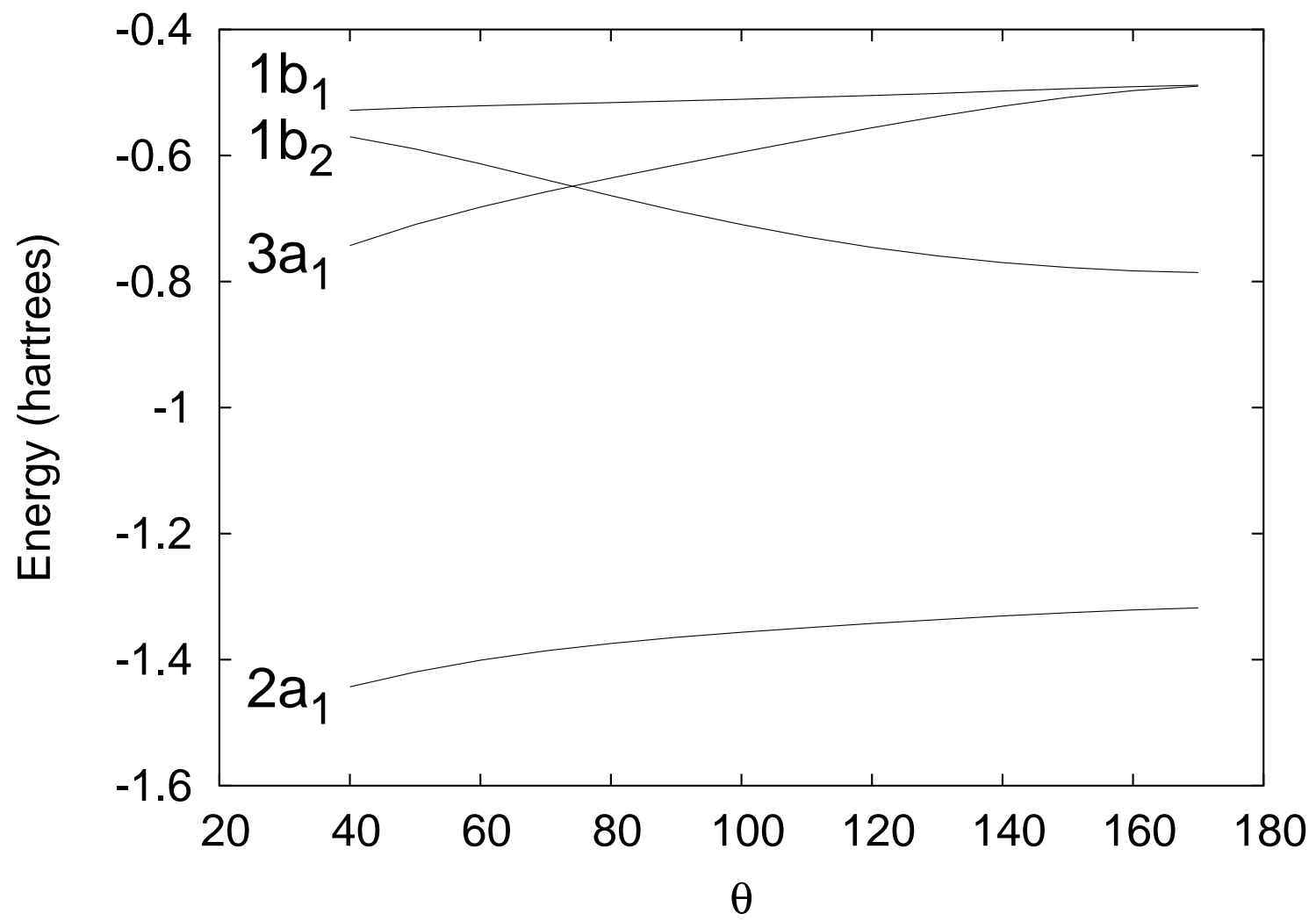

FIG. 3: Walsh diagram of SCF orbital energies of ground state $\mathrm{H}_{2} \mathrm{O}$ at $r_{1}=r_{2}=1.81 a_{0}$, as a function of the bond angle $\theta$.

since the ${ }^{2} A_{1}$ resonance state is characterized by a hole in the $3 a_{1}$ orbital, its energy increases as the bond angle is decreased, and that of the ${ }^{2} B_{2}$ resonance decreases.

As a result, the real parts of the energies of these resonances will cross. Because they have different couplings to the continuum, their imaginary parts need not be equal in $\mathrm{C}_{2 v}$ symmetry, and therefore the crossing of the real parts does not exactly correspond to an acutal degeneracy of these states. Our calculations place the crossing of the real parts at a bond angle of approximately $73^{\circ}$ when the $\mathrm{OH}$ bond distance is $1.81 a_{0}$. In Fig 4 we show the potential curves obtained from four-configuration MCSCF calculations on each state that included the three excitations $4 a_{1}^{2} \rightarrow 2 b_{2}^{2}, 5 a_{1}^{2}, 2 b_{1}^{2}$, which are the dominant correlating configurations at the equilibrium geometry of the neutral. In this figure we see the crossing of the real parts of the ${ }^{2} A_{1}$ and ${ }^{2} B_{2}$ energies and also the $\Pi$ degeneracy at $\mathrm{H}-\mathrm{O}-\mathrm{H}$ linear geometry between the ${ }^{2} A^{\prime \prime}\left({ }^{2} B_{1}\right)$ and $1{ }^{2} A^{\prime}\left({ }^{2} A_{1}\right)$ states, which will lead to Renner-Teller coupling between them. 


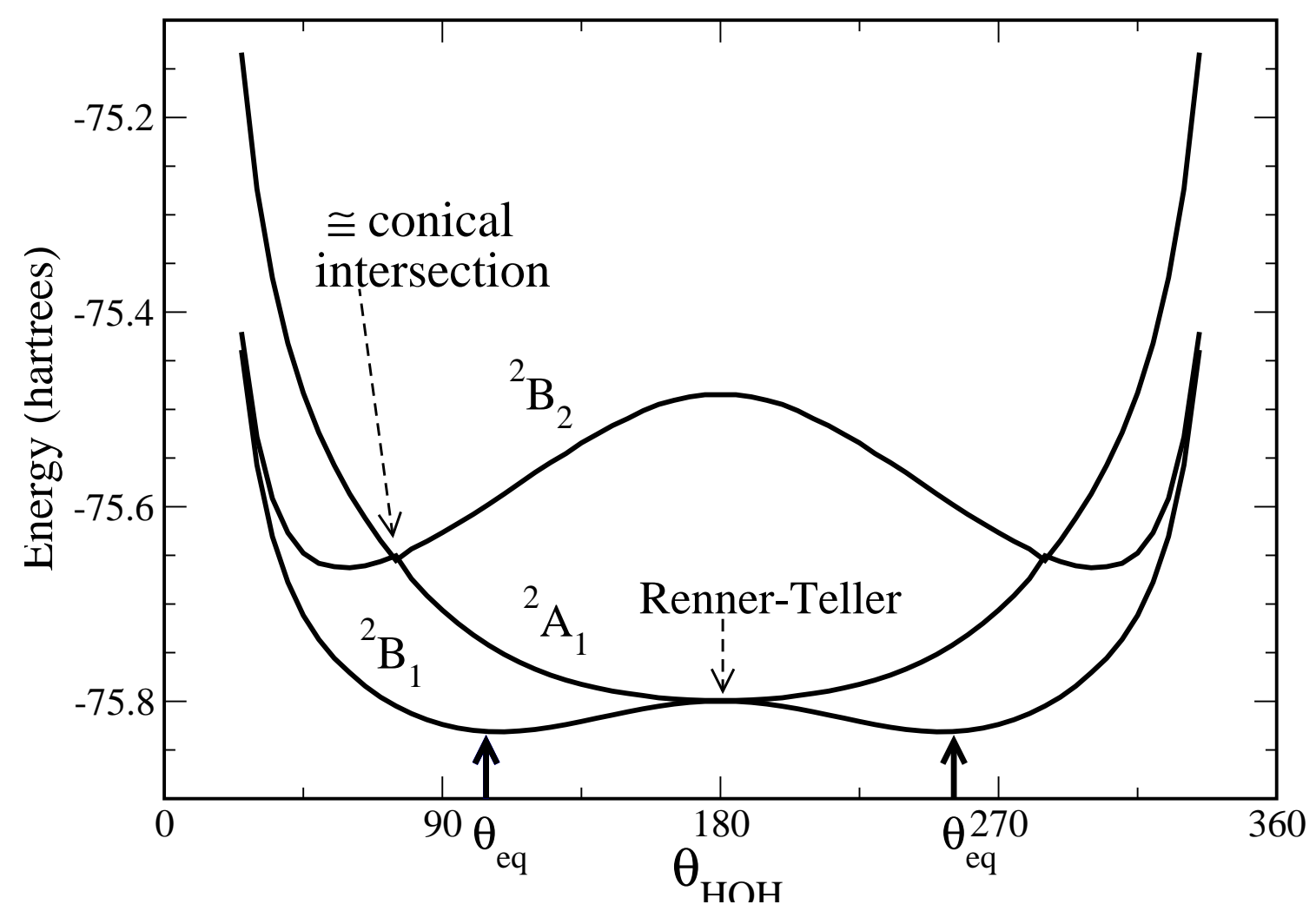

FIG. 4: Real parts of resonance energies for $\mathrm{OH}$ bond distance $=1.81 a_{0}$ in $\mathrm{C}_{2 v}$ geometry from the four-configuration MCSCF calculation described in text.

Distorting the molecule away from a point in $\mathrm{C}_{2 v}$ geometry where the real parts of the energies cross, it is possible, for a range of $\mathrm{OH}$ bond distances, to find a point where both the real and imaginary parts are equal, thereby locating an actual degeneracy between resonance states. For the ${ }^{2} A_{1}$ and ${ }^{2} B_{2}$ resonances this is indeed the case and the resulting conical intersection is analogous to the one between the $2{ }^{1} A_{1}$ and $1^{1} B_{2}$ states of neutral $\mathrm{H}_{2} \mathrm{O}$ which has been discussed elsewhere [32] in the context of photodissociation. However, in the present case we have a conical intersection of resonant states, not bound states. Conical intersections of metastable states have been discussed in the literature [33-35], and have analytical properties that are different from their bound-state counterparts.

To understand the case of a conical intersection between resonances, consider the simplified case of a two-dimensional electronic Hilbert space with $N$ nuclear degrees of freedom, 
collectively denoted $\vec{q}$, whose electronic Hamiltonian may be expressed

$$
\mathbf{H}(\vec{q})=\left(\begin{array}{ll}
H_{11}(\vec{q}) & H_{12}(\vec{q}) \\
H_{12}(\vec{q}) & H_{22}(\vec{q})
\end{array}\right)
$$

For a bound state system the matrix elements above may be taken to be real numbers, in which case the equation

$$
\operatorname{Det}\left(\mathbf{H}(\vec{q})-E_{i}(\vec{q}) \mathbf{I}\right)=0
$$

will yield degenerate eigenvalues $E_{1}$ and $E_{2}$ only if the two independent (real-valued) equalities,

$$
\begin{array}{r}
H_{11}=H_{22} \\
H_{12}=0
\end{array}
$$

are satisfied. If the adiabatic states belong to the same symmetry representation, then neither of these conditions are satisfied a priori, and a seam of intersection will have dimension $\mathrm{N}-2$, which is a conical intersection.

Whereas the energies of bound electronic states can generally be obtained as the eigenvalues of a real symmetric matrix, resonance energies may be found as eigenvalues of a complex symmetric matrix. There are several ways to see that this is the case, but perhaps the most direct is to consider the point spectrum of the Hamiltonian under complex scaling of its coordinates $[36,37]$ which maps onto the poles of the resolvent. The roots of a complex symmetric matrix $\mathbf{M}$,

$$
\mathbf{M}=\left(\begin{array}{ll}
A & B \\
B & D
\end{array}\right)
$$

will be degenerate if the two independent constraints

$$
\begin{aligned}
& \operatorname{Re}\left((A-D)^{2}+4 B^{2}\right)=0 \\
& \operatorname{Im}\left((A-D)^{2}+4 B^{2}\right)=0
\end{aligned}
$$

are satisfied, and so for resonances we will in general expect that degeneracies occur along seams of dimension $N-2$, just as in the bound state case for states of the same symmetry. Seams of intersection of the real parts of the eigenvalues of $\mathbf{M}$ will occur where Eq. (6) is satisfied and the inequality

$$
(A-D)^{2}+4 B^{2} \leq 0
$$


holds. For seams of intersection of the imaginary parts, this inequality is reversed. In general, such seams will occur along (possibly curvilinear) line segments which terminate at degenerate points. In our calculations we have found cases with both one and two such points of degeneracy and we give specific models for those situations below.

It is important to note that the existence of geometries of high symmetry, in which the two states belong to different irreducible representations and therefore $B=0$, still leaves two conditions to be satisfied in Eqs. (5) and (6). Therefore, whereas in bound-state theory there is a class of conical intersections which lie entirely within such high-symmetry geometries, there will be no such class of seams of degeneracy of metastable states. Instead, we may find the actual degeneracies slightly offset from these higher-symmetry geometries. That is indeed the case in our calculations of the conical intersection of the surfaces that correspond in $\mathrm{C}_{2 v}$ geometry to the ${ }^{2} A_{1}$ and ${ }^{2} B_{2}$ resonances.

Feuerbacher et al. [34] examined the effect of switching on the coupling of the resonance states to the continuum for some simple model problems, and they made several observations that are reflected in the results of our calculations. These authors observed that the effect of switching on the continuum coupling on a conical intersection is to split the single seam of degeneracy into two. The essential result, first demonstrated by Estrada et al. [33], is that the real part of the resonance energies behaves as in Fig. 5: there is a line segment along which the real part of the resonance energies are the same.

In Figure 5 are plotted the real parts of the energies of the resonances obtained from the "minimal model" of Feuerbacher et al. [34], which are solutions $E_{i}$ of the eigenvalue equation

$$
\operatorname{Det}\left(\begin{array}{cc}
x-i \Gamma_{1}-E_{i} & y \\
y & -x-i \Gamma_{2}-E_{i}
\end{array}\right)=0
$$

This model makes a prediction about the trajectories of the resonance poles $E_{i}$ in the complex $E$ plane as functions of the parameters $x$ and $y$. Along a closed path in this parameter space that encircles one of the branch points shown in Fig. 6, the resonance poles interchange positions. In Fig. 6 the closed path from A to B to C to D and back to A takes the system around one of the branch point degeneracies of the model. The behavior of the resonance energies in the complex plane is also shown in Fig. 6. Going from point B to C, the real parts of the resonance energies avoid one another, and the widths cross. Going from point $\mathrm{D}$ to $\mathrm{A}$, the real parts cross, while the imaginary parts avoid one another. The result 


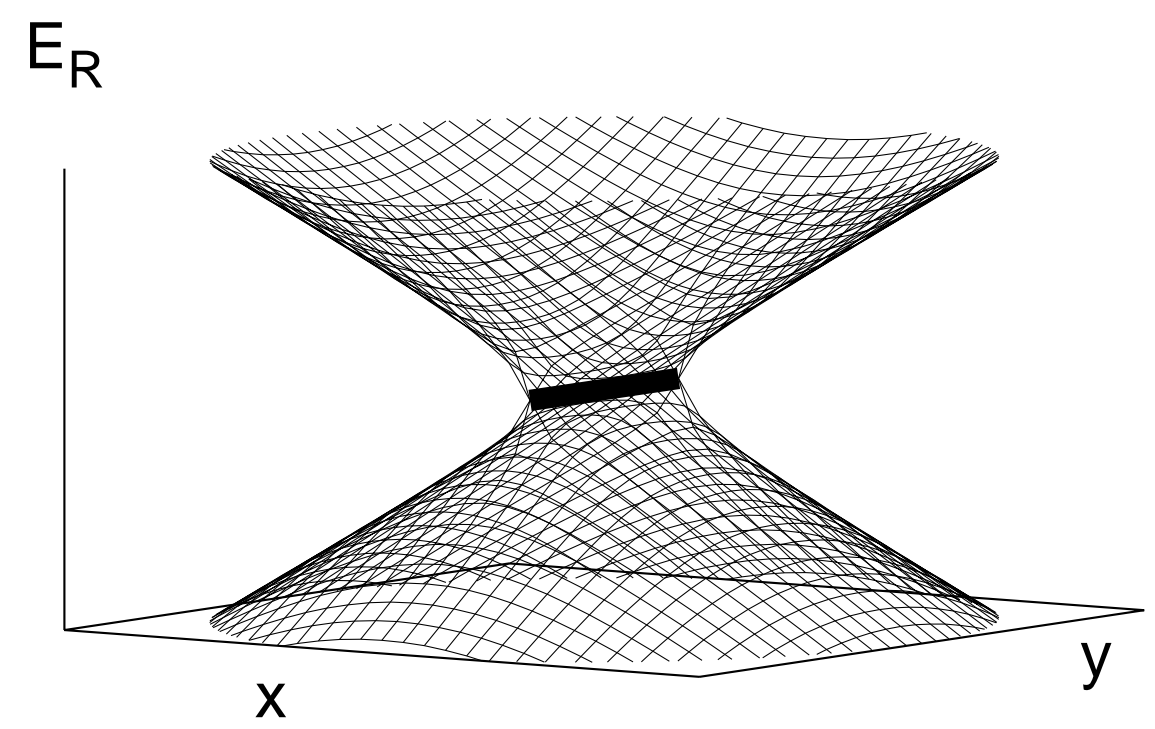

FIG. 5: Value of the real part of the energy of two interacting resonances, from "minimal model" of ref. [34].

is that, after following this closed loop in parameter space, the resonances have changed places.

This behavior is a reflection of the branch-point degeneracy in Eq. (8) which has been circumnavigated by this path. It has an interesing parallel in the theory of "hidden crossings" [38-40], in which the analytic continuation of bound-state potential energy curves into regions of complex-valued geometries often yields similar branch points. In this way it is seen that the various bound state potential energy curves are actually different branches of the same multivalued function in the complex coordinate plane. The pairing of the branch points in resonant potential energy surfaces which were observed in the complex symmetric matrix models of Ref. [34], and which we have found in calculations on the ${ }^{2} A_{1} /{ }^{2} B_{2}$ conical intersection, is similar to the pairing of hidden crossings with mirror-image pairs across the real coordinate axes. These similarities are not surprising, since the analytic continuation of a real symmetric bound-state Hamiltonian into the complex coordinate plane also yields 

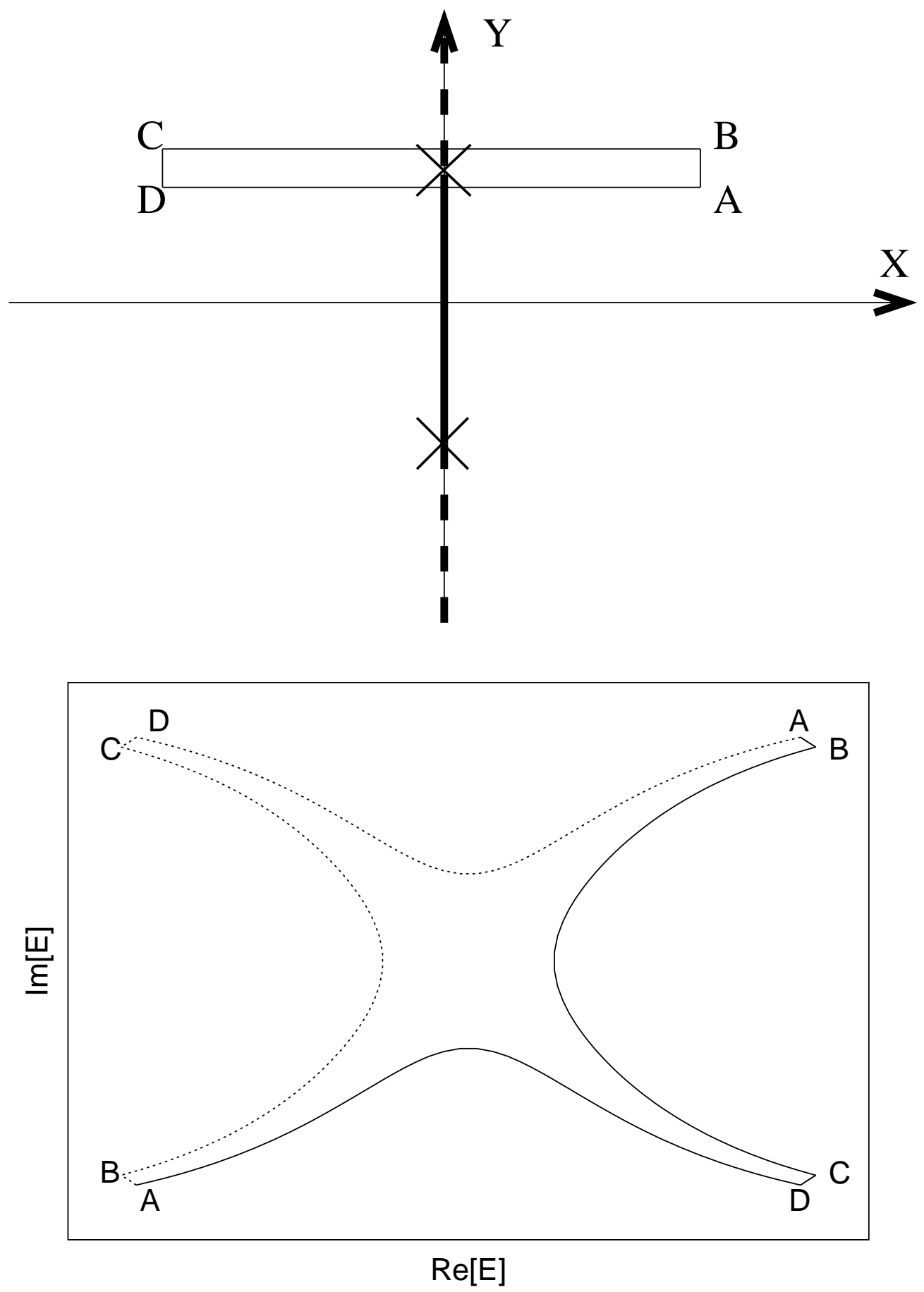

FIG. 6: Upper: Path in the $(x, y)$ plane for the eigenvalue trajectory in the lower panel. Degeneracies of model of Ref. [34] are noted with X's; the seam of intersection of the real parts of the resonance energies is denoted by a solid line, and the seam of intersection of the imaginary parts is denoted by a dashed line. Lower: Trajectory in the complex plane of eigenvalues $E_{1}$ (solid line) and $E_{2}$ (dashed line) as parameters $x$ and $y$ are varied according to upper panel from point $\mathrm{A} \rightarrow \mathrm{B} \rightarrow \mathrm{C} \rightarrow \mathrm{D} \rightarrow \mathrm{A}$. 

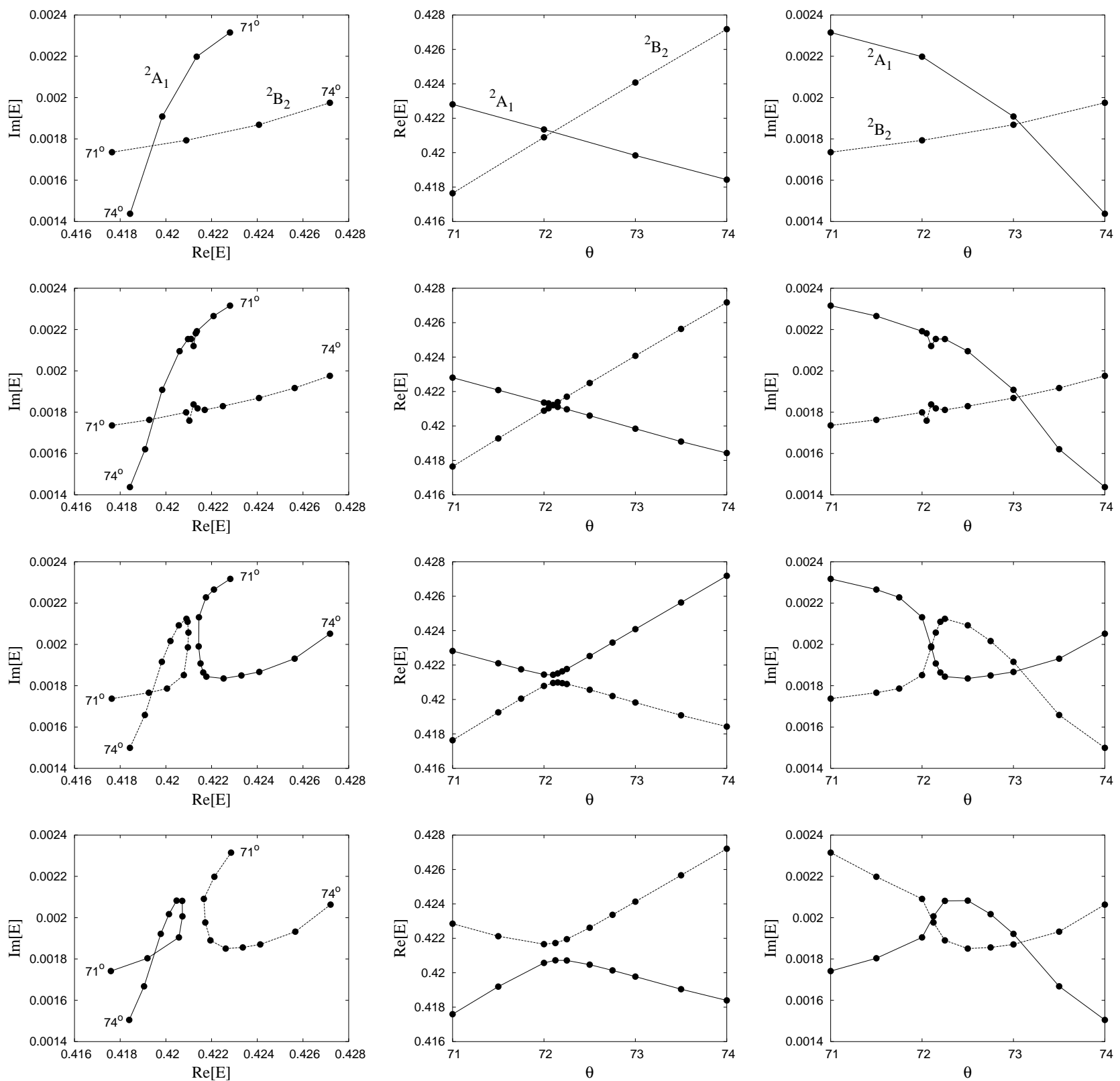

FIG. 7: Real and imaginary parts of ${ }^{2} A_{1}$ and ${ }^{2} B_{2}\left(1^{2} A^{\prime}\right.$ and $\left.2{ }^{2} A^{\prime}\right)$ energies, in hartrees, as a function of bond angle $\theta$, at (top row to bottom row) $r_{1}=r_{2}=1.81 a_{0} ; r_{1}=1.8094, r_{2}=1.8106$ $a_{0} ; r_{1}=1.8075, r_{2}=1.8125 a_{0} ; r_{1}=1.805, r_{2}=1.815 a_{0}$.

a complex symmetric matrix.

We found branch point behavior similar to that exhibited by the model problem above in our computed ${ }^{2} A_{1} /{ }^{2} B_{2}$ resonance energies. This behavior is manifested as a qualitative change in the dependence of the resonance energies on one nuclear coordinate as another 
is varied. The signature of the branch point is a transition from the case where the real parts of the resonance energy cross while the imaginary parts avoid as the resonance poles approach each other, to a case where the real parts avoid while the imaginary parts cross.

That transition can be seen in the panels of Fig. 7, which shows the complex energies of the $1^{2} A^{\prime \prime}$ and $2{ }^{2} A^{\prime \prime}$ resonances (obtained from complex Kohn variational scattering calculations described in Appendix B) plotted as a functions of nuclear geometry. Each of the four rows of that figure show the results of varying the bond angle for different values of one $\mathrm{OH}$ bond distance, $r_{1}$, while the other is fixed by the relation $\left(r_{1}+r_{2}\right) / 2=1.81 a_{0}$. The top row shows the variation of $\theta$ in $\mathrm{C}_{2 v}$ symmetry and we see the real parts of the ${ }^{2} A_{1}$ and ${ }^{2} B_{2}$ potentials cross around $\theta=72$ degrees while the imaginary parts cross at a different geometry, around $\theta=73$ degrees. Thus, at $r_{1}=r_{2}=1.81 a_{0}$, there is no degeneracy between the ${ }^{2} A_{1}$ and ${ }^{2} B_{2}$ states for any $\theta$.

To find the actual branch point degeneracy, we must break $\mathrm{C}_{2 v}$ symmetry. The next row in Fig. 7 , at $r_{1}=1.8094, r_{2}=1.8106$, shows that the connectivity of trajectories in the first row is maintained, and we see a suggestion of the imaginary parts avoiding each other near the angle where the real parts cross. Up to this point the states are most aptly labeled " $\mathrm{A}_{1}$ like" and "B $\mathrm{B}_{2}$-like", not "upper" and "lower." However, by the third row, at $r_{1}=1.8075$, $r_{2}=1.8125$, the connectivity has changed and now the real parts of the resonance energy avoid one another as $\theta$ is varied, as is the case for the model problem along path $\mathrm{BC}$ in Fig. 6 .

Therefore, at some point between the geometries of the second and third row, an actual degeneracy of the resonant states was passed. A fit of the quantity $\left|E_{1}-E_{2}\right|^{2}$ evaluated at 15 points near these geometries to a quadratic form yielded $\left(r_{1}, r_{2}, \theta\right)=\left(1.8089 a_{0}, 1.8111 a_{0}\right.$, $72.103^{\circ}$ ) for the location of the conical intersection at $r_{1}+r_{2}=3.62 a_{0}$. A mirror-image degenerate seam, of course, passes through $r_{1}=1.8111, r_{2}=1.8089$.

Because these two mirror-image seams are so close - much closer than the de Broglie wavelength for the moving nuclei - we do not expect that the two-seam structure of this conical intersection of metastable states will have any implications for the nuclear dynamics. In other words, we expect that we may treat this conical intersection using the standard array of bound-state techniques, approximating the conical intersection as a single seam, without compromising the subsequent description of the nuclear dynamics near the intersection. 


\section{B. ${ }^{2} A_{1}\left(1^{2} A^{\prime}\right)$ state}

As explained above, starting from the equilibrium geometry of neutral water, the energy of the ${ }^{2} A_{1}$ anion decreases as the $\mathrm{H}-\mathrm{O}-\mathrm{H}$ bond angle increases. At linear $\mathrm{H}-\mathrm{O}-\mathrm{H}$ geometry, the ${ }^{2} A_{1}\left(1^{2} A^{\prime}\right)$ state and ${ }^{2} B_{1}\left({ }^{2} A^{\prime \prime}\right)$ state are the two degenerate components of a ${ }^{2} \Pi$ state. Thus, if it is first brought to linear $\mathrm{H}-\mathrm{O}-\mathrm{H}$ geometry and then dissociated, the ${ }^{2} A_{1}\left(1^{2} A^{\prime \prime}\right)$ state correlates with $\mathrm{H}^{-}+\mathrm{OH}\left({ }^{2} \Pi\right)$, as does the ${ }^{2} A^{\prime \prime}$ state. In turn, if the $\mathrm{OH}$ fragment is then dissociated, the same charge-transfer behavior as demonstrated in Section III for the ${ }^{2} A^{\prime \prime}$ state will occur. Therefore, by this mechanism the ${ }^{2} A_{1}$ state can correlate with the three-body breakup channel $\mathrm{O}^{-}+\mathrm{H}+\mathrm{H}$, in agreement with the results of Curtis and Walker [12] who found evidence of this three-body channel from dissociative attachment to the ${ }^{2} A_{1}$ state.

The ${ }^{2} A_{1}$ state may also be dissociated directly to the three-body breakup region while maintaining $\mathrm{C}_{2 v}$ geometry. We have performed scattering calculations along several such paths, which demonstrate that the ${ }^{2} A_{1}$ state does indeed correlate to $\mathrm{O}^{-}+\mathrm{H}+\mathrm{H}$ as the state is brought directly to this arrangement. Moreover, those calculations show that the correct asymptote in the $\mathrm{O}+\mathrm{H}_{2}$ two-body breakup arrangement is $\mathrm{O}^{-}+\mathrm{H}_{2}\left(1 \sigma_{g} 1 \sigma_{u}\right)$ for the ${ }^{2} A_{1}$ resonance. Thus we find that the ${ }^{2} A_{1}$ Feshbach resonance does not correlate to the lowest ${ }^{2} A_{1}$ state of the anion in that arrangement, which is $\mathrm{O}^{-}+\mathrm{H}_{2}\left(1 \sigma_{g}^{2}\right)$.

We provide evidence for our assignment of the $\mathrm{O}^{-}+\mathrm{H}_{2}\left(1 \sigma_{g} 1 \sigma_{u}\right)$ asymptote in Fig. 8 . This figure shows the real part of the energy of the resonant state as determined by scattering calculations where it is unbound, and by structure calculations where it is bound. The details of these calculations are included in Appendix B; for the present we will note only that they are performed with a full CI representation of the target states in an atomic orbital basis. In Fig. 8 we dissociate the resonance from a geometry $\left(r_{1}=r_{2}=2.0 a_{0}, \theta=104.5^{\circ}\right)$ slightly stretched from the equilibrium geometry of the neutral, by increasing the distance between the oxygen nucleus and the $\mathrm{H}_{2}$ center of mass and keeping the $\mathrm{H}_{2}$ internuclear distance constant at $3.16 a_{0}$. This bond length is just big enough to bind the $\mathrm{O}^{-}+\mathrm{H}_{2}$ $\left(1 \sigma_{g} 1 \sigma_{u}\right)$ state as per the current treatment. Plotted in this figure is the energy of the ${ }^{2} A_{1}$ Feshbach resonance where bound and unbound, and the lowest ${ }^{2} A_{1}$ state of the anion, where bound. As is clear from this figure, the ${ }^{2} A_{1}$ Feshbach resonance correlates not to $\mathrm{O}^{-}+\mathrm{H}_{2}$ $\left(1 \sigma_{g}^{2}\right)$, but to $\mathrm{O}^{-}+\mathrm{H}_{2}\left(1 \sigma_{g} 1 \sigma_{u}\right)$. We have found the same correlation along different paths, 


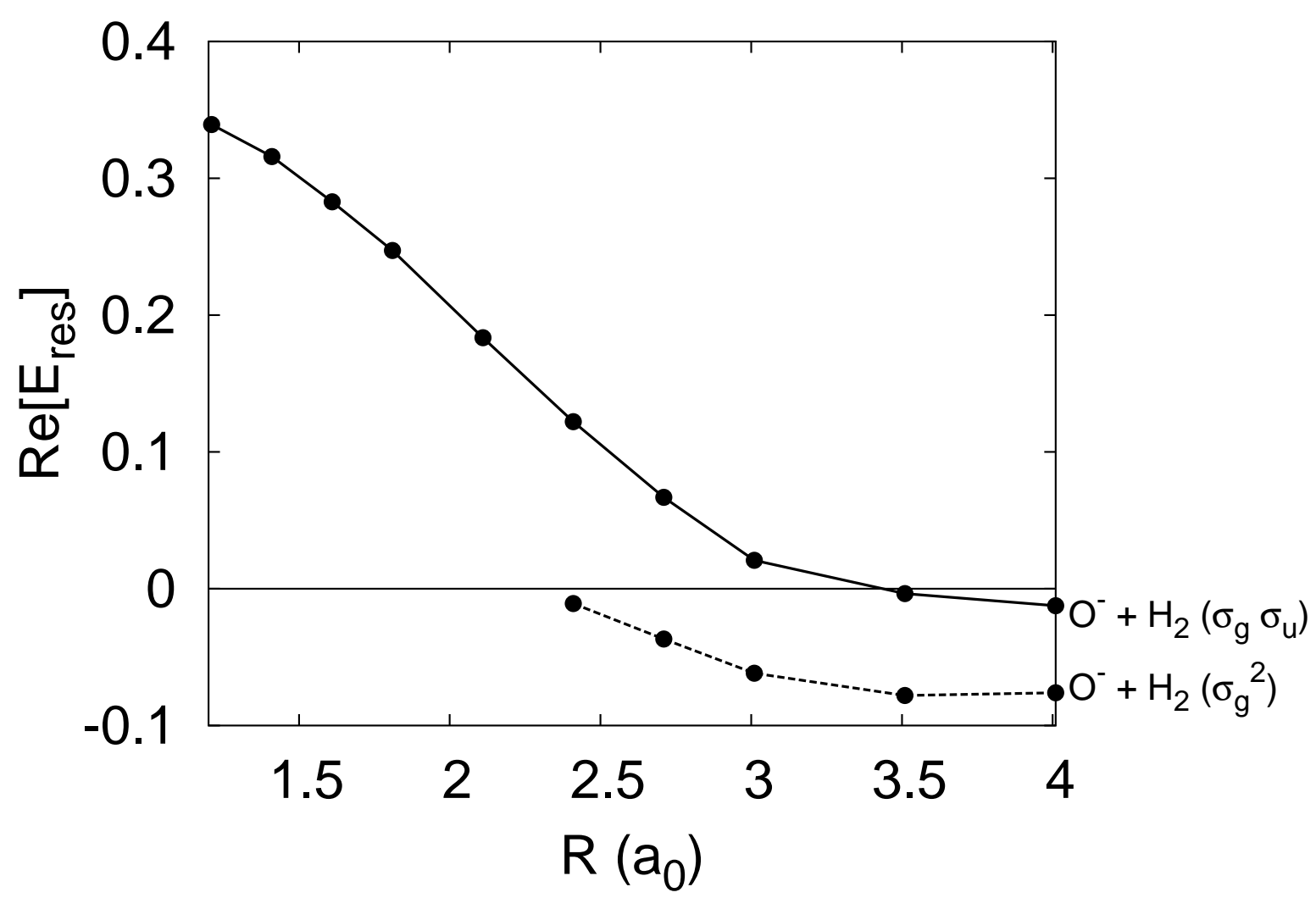

FIG. 8: Real part of energy of the ${ }^{2} A_{1}$ Feshbach resonance, where bound $(<0)$ and unbound ( $>0$ ), and lowest ${ }^{2} A_{1}$ state of the anion, where bound, as a function of the distance $R$ between the oxygen nucleus and the $\mathrm{H}_{2}$ center of mass, in $\mathrm{C}_{2 v}$ geometries, with $\mathrm{r}_{H H}=3.16 a_{0}$.

including the path from the equilibrium geometry of the neutral to the squeezed geometry at $r_{1}=r_{2}=1.81 a_{0}, \theta=45^{\circ}\left(r_{H H}=1.40\right)$ to dissociation. Thus it appears that the ${ }^{2} A_{1}$ Feshbach resonance correlates globally to this asymptote.

This finding is reassuring in light of the experiments on associative detachment. The experiments on associative detachment of $\mathrm{O}^{-}+\mathrm{H}_{2}[26,28,30,31]$ and $\mathrm{OH}^{-}+\mathrm{H}[27,29]$ indicate that these fragments correlate with an attractive state which has a very large width at highly distorted geometries of neutral $\mathrm{H}_{2} \mathrm{O}$ - a width much larger than we would expect for the ${ }^{2} A_{1}$ Feshbach resonance. This state, with configuration $\left[\mathrm{H}_{2} \mathrm{O}\right] 4 a_{1}^{1}$, has been termed a shape resonance $[41,42]$, although it is surely extremely broad at the equilibrium geometry of the neutral. It is clear from our scattering calculations that this ${ }^{2} A_{1}$ "shape resonance" is distinct from the ${ }^{2} A_{1}$ Feshbach resonance relevant to dissociative attachment, and that these two states represent separate poles of the S-matrix. The ${ }^{2} A_{1}$ "shape resonance" is 


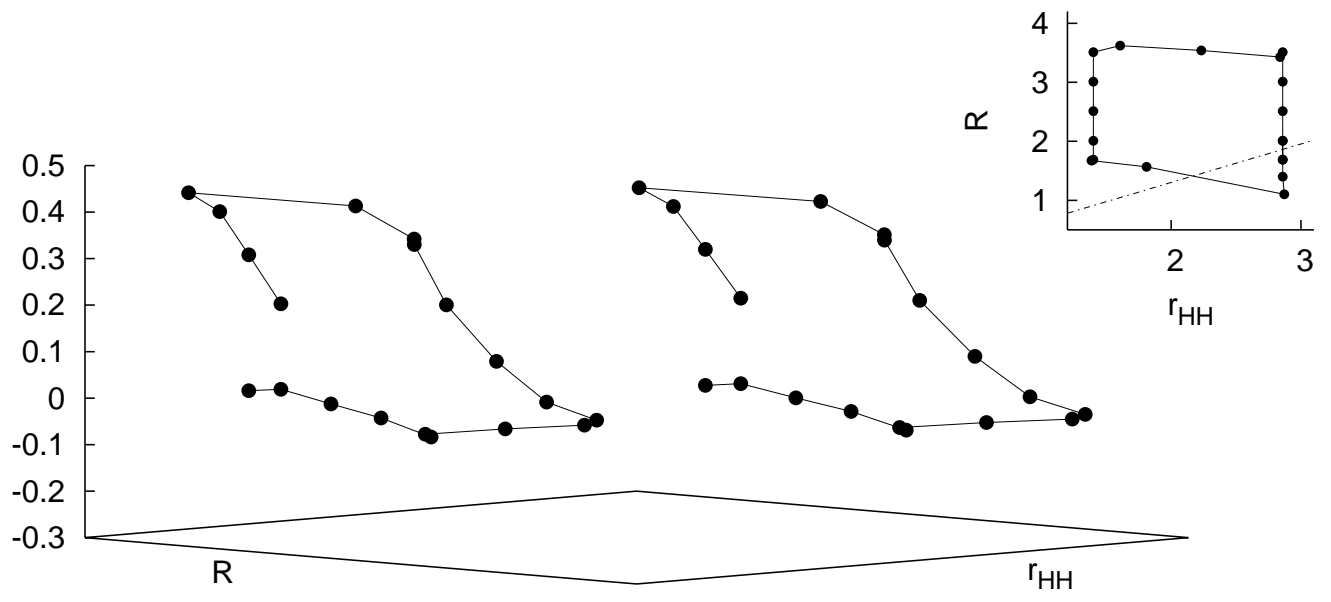

FIG. 9: Real parts of energies of ${ }^{2} B_{2}$ states in $\mathrm{C}_{2 v}$ geometries, in hartrees above the ground state energy, as a function of $\mathrm{H}-\mathrm{H}$ bond distance $r_{H H}$ and the distance between the $\mathrm{H}_{2}$ center of mass and the oxygen nucleus, $R$. Inset: top view including a line at $\theta=73^{\circ}$. Left image is aligned with the printed axes; right image is its stereographic partner for 3D viewing.

most likely irrelevant to the dissociative attachment process.

\section{C. ${ }^{2} B_{2}\left(2^{2} A^{\prime}\right)$ state}

The experiments of Curtis and Walker [12] indicated that $\mathrm{OH}\left({ }^{2} \Sigma\right)+\mathrm{H}^{-}$, i.e. electronically excited $\mathrm{OH}$, is produced from the third resonance peak. Since the other two resonance states, $1^{2} A^{\prime}$ and ${ }^{2} A^{\prime \prime}$, correlate with the two components of ground state $\mathrm{OH}\left({ }^{2} \Pi\right)+\mathrm{H}^{-}$ state in that arrangement, the $2{ }^{2} A^{\prime}$ state is left to correlate to $\mathrm{H}^{-}+\mathrm{OH}\left({ }^{2} \Sigma\right)$. This asymptote can also be expected for this resonance because the $1 b_{2}$ SCF orbital becomes the sigma bonding molecular orbital on $\mathrm{OH}$. We have confirmed this asymptote in electron scattering calculations using the complex Kohn method.

We now turn to the behavior of the ${ }^{2} B_{2}$ resonance within $\mathrm{C}_{2} v$ geometries. Within this cut of the potential surface the ${ }^{2} B_{2}$ state is distinct from the ${ }^{2} A_{1}$ state. We have found that the ${ }^{2} B_{2}$ state has a seemingly isolated branch-point degeneracy with a ${ }^{2} B_{2}$ shape resonance. This branch point leads to an inherent double-valuedness of the ${ }^{2} B_{2}$ surface within $\mathrm{C}_{2 v}$ 
geometries.

The ${ }^{2} B_{2}$ shape resonance, with configuration $\left[\mathrm{H}_{2} \mathrm{O}\right] 2 b_{2}{ }^{1}$, has been observed in experiments and calculations on the process of electron-impact vibrational excitation of water [41-47]. This resonance is found near $7 \mathrm{eV}$ incident energy and is several $\mathrm{eV}$ wide at the equilibrium geometry of the neutral; it is too wide for the present theoretical investigation to locate at that geometry. However, we have located two resonance features in ${ }^{2} B_{2}$ symmetry at certain distorted geometries, and we have no plausible candidate besides the ${ }^{2} B_{2}$ shape resonance to be responsible for the second feature.

We have performed scattering calculations in ${ }^{2} B_{2}$ symmetry within $\mathrm{C}_{2 v}$ geometries. These calculations are described in Appendix B and are exactly analagous to the calculations used to determine the asymptotes of the ${ }^{2} A_{1}$ state: they define the target states and 11-electron configurations responsible for the resonance features by full CI in an atomic orbital basis. We have performed analagous CI structure calculations in the regions where the resonance becomes bound.

These calculations demonstrate the inherent double valuedness of this potential surface and indicate that the ${ }^{2} B_{2}$ Feshbach resonance can correlate to either of two three-body breakup channels. Starting from the equilibrium geometry of neutral $\mathrm{H}_{2} \mathrm{O}\left(r_{1}=r_{2}=1.81\right.$ $a_{0}, \theta=104.5^{\circ}$ ), if the bond angle is first decreased to $45^{\circ}$, corresponding to $r_{H H}=1.4 a_{0}$, and then the $\mathrm{H}_{2}$ fragment is dissociated from the oxygen, we obtain the bound two-body asymptote $\mathrm{O}^{-}+\mathrm{H}_{2}$. The $\mathrm{H}_{2}$ fragment may then be dissociated to produce $\mathrm{O}^{-}+\mathrm{H}+\mathrm{H}$.

In contrast, if the bond angle is kept constant at $104.5^{\circ}$ and the hydrogens are dissociated symmetrically, the ${ }^{2} B_{2}$ Feshbach resonance does not correlate to $\mathrm{O}^{-}+\mathrm{H}+\mathrm{H}$. It stays higher in energy and appears to correlate with the state $\mathrm{O}\left({ }^{1} D\right)+\mathrm{H}+\mathrm{H}^{-}$.

These result are plotted in the stereogram in Fig. 9, in which the location of the ${ }^{2} B_{2}$ resonance in $\mathrm{C}_{2 v}$ geometries is plotted as a function of the $\mathrm{H}-\mathrm{H}$ bond distance, $r_{H H}$, and the distance between the $\mathrm{H}_{2}$ center of mass and the oxygen nucleus, $R$. The double-valuedness of the ${ }^{2} B_{2}$ state is clear from this figure. The geometries $r_{H H}=2.86, R=1.69$ and $r_{H H}=2.86, R=2.01$, which are the first two and last two points on the path, have two resonance locations plotted. The exact path in the $\mathrm{C}_{2 v}$ plane is shown at upper right. Going clockwise, the ${ }^{2} B_{2}$ Feshbach resonance becomes bound as $\mathrm{O}^{-}+\mathrm{H}_{2}$ between $r_{H H}=1.40$, $R=2.51$ and $r_{H H}=1.40, R=3.01$, and stays so until $r_{H H}=2.86, R=2.51$, at which point it has become the ${ }^{2} B_{2}$ shape resonance. Also plotted is the line $\theta=73^{\circ}$, which approximates 
the location of the ${ }^{2} A_{1} /{ }^{2} B_{2}$ conical intersection.

The three-body asymptote $\mathrm{O}\left({ }^{1} D\right)+\mathrm{H}+\mathrm{H}^{-}$is consistent with the two-body asymptote $\mathrm{OH}\left({ }^{2} \Sigma\right)+\mathrm{H}^{-}$, but not $\mathrm{H}_{2}+\mathrm{O}^{-}$. Following the $\mathrm{H}_{2}+\mathrm{O}^{-}$asymptote into the three-body breakup region, we obtain $\mathrm{H}+\mathrm{H}+\mathrm{O}^{-}$. In so doing, we have followed the ground state of the system, and therefore there can be no charge-transfer crossing of the type demonstrated above for the $\mathrm{OH}\left({ }^{2} \Pi\right)+\mathrm{H}^{-}$asymptote of the ${ }^{2} B_{1}$ and ${ }^{2} A_{1}$ states to account for this inconsistency. Therefore, the ${ }^{2} B_{2}$ surface is inherently double-valued.

It is useful to have a simple mathematical model for this behavior. None of the models of Refs. [33-35] produce an isolated degeneracy. In contrast, the following $2 \times 2$ complex symmetric eigenvalue equation,

$$
\operatorname{Det}\left(\begin{array}{cc}
x-2 i \exp (-y)-E_{i} & y \\
y & -x-i-E_{i}
\end{array}\right)=0
$$

produces a single point of intersection at $(x, y)=(0,0.2662)$. The real parts of the eigenvalues $E_{i}$ of Eq. (9) are plotted in Figure 10. When the point of degeneracy is circumnavigated, the two resonances exchange identities, just as in Fig. 6.

The difference between the model in Eq. (9) and the one in Eq. (8) is that instead of having fixed widths for the noninteracting resonances, $\Gamma_{1}$ and $\Gamma_{2}$, Eq. (9) has one width fixed $\left(\Gamma_{2}=1\right)$ while the other varies with $\mathrm{y}$. A variety of behaviors can be seen in such a model depending on the parametrization of $\Gamma_{1}$ and $\Gamma_{2}$, including one, two or three points of degeneracy between the resonance eigenvalues. Presumably, all of those behaviors might be found in real systems, although thus far in $\mathrm{H}_{2} \mathrm{O}^{-}$we have found only the two types we have discussed here, having either one or two branch point degeneracies.

\section{CONCLUSION}

We have performed calculations which have determined the bulk of the features of the adiabatic surfaces for the resonant states of $\mathrm{H}_{2} \mathrm{O}^{-}$relevant to the dissociative attachment process. To summarize some of the findings we have presented, we list the asymptotes of the resonant states of $\mathrm{H}_{2} \mathrm{O}^{-}$in Table $\mathrm{I}$.

We have to take account of the fact that the combination of the ${ }^{2} A_{1} /{ }^{2} B_{2}$ conical intersection between Feshbach resonances and the seam of degeneracy between the ${ }^{2} B_{2}$ Feshbach 


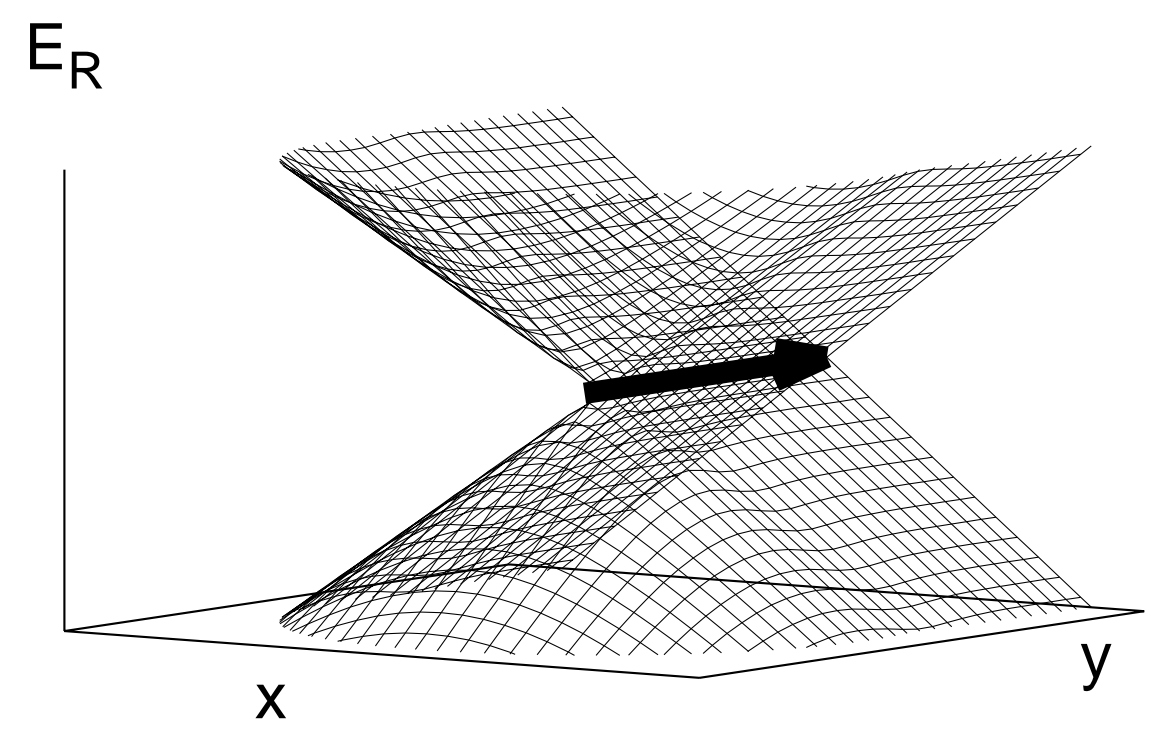

FIG. 10: Real part of energies of resonances in the single-intersection model of Eq. (9). Arrow indicates seam of degeneracy continues in that direction.

TABLE I: Asymptotes of resonant states. The symmetry of the states in $\mathrm{C}_{2 v}$ geometry is noted for the applicable asymptotes.

\begin{tabular}{|c|c|c|c|}
\hline & $\mathrm{O}-\mathrm{H}_{2}$ & $\mathrm{OH}-\mathrm{H}$ & $\mathrm{O}-\mathrm{H}-\mathrm{H}$ (three-body) \\
\hline${ }^{2} A^{\prime \prime}$ & $\mathrm{O}^{-}\left(\mathrm{X}^{2} P\right)+\mathrm{H}_{2}\left(\mathrm{X} 1 \sigma_{g}^{2}\right)$ & $\mathrm{OH}\left(\mathrm{X}^{2} \Pi\right)+\mathrm{H}^{-}$ & $\mathrm{O}^{-}\left(\mathrm{X}^{2} P\right)+\mathrm{H}+\mathrm{H} \quad\left(B_{1}\right)$ \\
\hline $1^{2} A^{\prime}$ & $\mathrm{O}^{-}\left(\mathrm{X}^{2} \mathrm{P}\right)+\mathrm{H}_{2}\left(\mathrm{X} 1 \sigma_{g}^{2}\right)$ & $\mathrm{OH}\left(\mathrm{X}^{2} \Pi\right)+\mathrm{H}^{-}$ & $\mathrm{O}^{-}\left(\mathrm{X}^{2} P\right)+\mathrm{H}+\mathrm{H} \quad\left(A_{1}\right)$ \\
\hline & $A N D \quad \mathrm{O}\left({ }^{1} D\right)+\mathrm{H}_{2}^{-} \quad\left(B_{2}\right)$ & $A N D ?$ & $\mathrm{O}^{-}\left(\mathrm{X}^{2} P\right)+\mathrm{H}+\mathrm{H} \quad\left(B_{2}\right)$ \\
\hline $2^{2} A^{\prime}$ & $\mathrm{O}^{-}\left(\mathrm{X}^{2} P\right)+\mathrm{H}_{2}\left(1 \sigma_{g} 1 \sigma_{u}\right)$ & $\mathrm{OH}\left({ }^{2} \Sigma\right)+\mathrm{H}^{-}$ & $\mathrm{O}\left({ }^{1} D\right)+\mathrm{H}^{-}+\mathrm{H} \quad\left(B_{2}\right)$ \\
\hline
\end{tabular}

and ${ }^{2} B_{2}$ shape resonance can lead to either of two topological cases, because all three surfaces are connected. The ${ }^{2} A_{1} /{ }^{2} B_{2}$ conical intersection produces a "lower cone" and "upper cone" that we have called the $1^{2} A^{\prime}$ and $2^{2} A^{\prime}$ states, respectively. The seam of degeneracy between the ${ }^{2} B_{2}$ Feshbach and ${ }^{2} B_{2}$ shape resonances could occur on either, and the present calculations indicate that it is most likely on the lower of the two surfaces, i.e., the $1^{2} A^{\prime}$ 
state (see inset of Fig. 7). So it appears that the $1^{2} A^{\prime}$ state is the one that is inherently double-valued in these calculations. For this reason in Table I there are two entries for the $1^{2} A^{\prime}$ state in each column.

We note then that there is some uncertainty in several of the entries in this table. A more accurate calculation could place seam of degeneracy of the ${ }^{2} B_{2}$ shape/Feshbach resonance states on the $2^{2} A^{\prime}$ state (upper cone). Secondly, we have not determined to which resonant state of $\mathrm{H}_{2}^{-}$the higher-energy ${ }^{2} B_{2}$ resonance correlates, nor have we examined the $\mathrm{OH}+\mathrm{H}$ asymptote of the ${ }^{2} B_{2}$ shape resonance. Nonetheless, we can make several comments about how the results of this study bear on the question of the dynamics of dissociative attachment, and how they may be related to the presently available experimental results.

First, it is observed that $\mathrm{O}^{-}+\mathrm{H}_{2}$ is by far the dominant product of dissociative attachment to the $2{ }^{2} A^{\prime}\left({ }^{2} B_{2}\right)$ state. However, this channel is not present as an asymptote of the $2^{2} A^{\prime}$ surface. Therefore, these products must result from nonadiabatic coupling between the $2^{2} A^{\prime}$ surface and the $1^{2} A^{\prime}$ surface, i.e. by dynamics following the ${ }^{2} B_{2}$ surface in $\mathrm{C}_{2 v}$ geometries. This is the consequence of the ${ }^{2} B_{2} /{ }^{2} A_{1}$ conical intersection that occurs slightly off of $C_{2 v}$ symmetry. By following the ${ }^{2} B_{2}$ state downhill along its gradient from the equilibrium geometry of the neutral to smaller bond angles, the $1^{2} A^{\prime}$ surface is easily reached (see Fig. 4). The nuclear dynamics leading to these products from the $2{ }^{2} A^{\prime}$ surface are therefore likely to follow this direct, symmetric dissociation path. So the peak in dissociative attachment generally identified as as the ${ }^{2} B_{2}$ Feshbach resonance, seems to be able to produce $\mathrm{O}^{-}$only because of this conical intersection.

Second, $\mathrm{O}^{-}+\mathrm{H}_{2}$ has been observed as a product of dissociative attachment via the ${ }^{2} A_{1}$ $\left(1^{2} A^{\prime}\right)$ resonance. However, the most direct path to these products goes uphill in energy for this state. Therefore, two other paths are likely to be involved. Production of $\mathrm{O}^{-}+\mathrm{H}_{2}$ from the ${ }^{2} A_{1}\left(1^{2} A^{\prime}\right)$ resonance can be due to Renner-Teller coupling to the ${ }^{2} A^{\prime \prime}$ state as indicated in Fig. 4. It can also be due to nuclear dynamics involving breaking $\mathrm{C}_{2 v}$ symmetry and traversing around the ${ }^{2} A_{1} /{ }^{2} B_{2}$ conical intersection, in which case the $\mathrm{O}^{-}+\mathrm{H}_{2}$ asymptote of the ${ }^{2} B_{2}$ state would be reached. Both of these mechanisms would probably involve the water molecule first straightening to linear $\left(\theta=180^{\circ}\right) \mathrm{H}-\mathrm{O}-\mathrm{H}$ geometry, and then continuing beyond $180^{\circ}$ to reach the $\mathrm{O}^{-}+\mathrm{H}_{2}$ well of either of the ${ }^{2} A^{\prime \prime}$ or $1^{2} A^{\prime}$ states.

The conclusions at which we have arrived in this paper, relating to the correct asymptotes of these states, dictate many requirements of an optimal orbital basis and configuration- 
interaction calculation. In addition, the double-valued ${ }^{2} B_{2}$ surface provides an interesting diabatization problem, which must be treated along with the more standard diabatization

of the ${ }^{2} A_{1} /{ }^{2} B_{2}$ conical intersection. The results of larger-scale calculations providing quantitative potential energy surfaces for these resonances, suitable for dynamics calculations, will be reported in a subsequent publication.

We close by noting that this study has indicated that a full theoretical treatment of the problem of dissociative attachment to water, incorporating all of the features of the electronic states described in this paper, may be prohibitively complicated. In all, there are eight distinct seams of degeneracy which we have identified among these resonant states: two mirror-image seams each for the charge transfer-behavior of the $\mathrm{OH}\left({ }^{2} \Pi\right)+\mathrm{H}^{-}$asymptote of the ${ }^{2} A^{\prime \prime}$ and $1^{2} A^{\prime}$ states; the degeneracy of the ${ }^{2} A^{\prime \prime}$ and $1^{2} A^{\prime}$ states at linear $\mathrm{H}-\mathrm{O}-\mathrm{H}$ geometry; the ${ }^{2} A_{1} /{ }^{2} B_{2}$ conical intersection, which actually comprises two mirror-image seams of degeneracy slightly displaced to either side of the $\mathrm{C}_{2 v}$ plane; and the ${ }^{2} B_{2}$ shapeFeshbach degeneracy.

\section{APPENDIX A: DETAILS OF OF BOUND STATE CALCULATIONS}

The resultss shown in Fig. 2 are the result of full CI calculations in an orthogonalized atomic orbital basis. We first obtained an atomic orbital space from calculations on the ionic $\mathrm{H}^{-}$and $\mathrm{O}^{-}$fragments. On $\mathrm{O}^{-}$, we performed a state-averaged $\mathrm{SCF}$ on the three spatial components of the $\mathrm{O}^{-}\left({ }^{2} \mathrm{P}\right)$ state, in order to obtain a symmetric atomic orbital basis. On the hydride state, we performed a multiconfiguration self-consistent field (MCSCF) calculation defined by full CI with two s orbitals. For these calculations we used the primitive basis of Gil et al. [16] augmented by diffuse orbitals with Gaussian exponents of 0.08 and $0.0333(s)$, and 0.2 and $0.05(p)$ on the hydrogens. These calculations yielded $1 \mathrm{~s}, 2 \mathrm{~s}, 2 \mathrm{p}_{x}, 2 \mathrm{p}_{y}$, and $2 \mathrm{p}_{z}$ orbitals on the $\mathrm{O}^{-}$, plus $1 \mathrm{~s}$ and $2 \mathrm{~s}$ orbitals on each of two H's. These comprise 9 orbitals and we have 11 electrons in the full problem for a total of 6048 configurations in the full CI calculation on the anion states.

This full CI treatment is designed to reproduce the correct ordering of the relevant states and to employ the smallest orbital basis possible. The correct ordering is necessary so that crossings of diabatic states are neither artificially created nor omitted, and the small orbital basis is useful in simplifying the spectrum when the relevant anionic species become 
unbound. The full CI treatment using these orbitals gives a small $\mathrm{H}^{-}$electron affinity of about $0.3 \mathrm{eV}$ and an electron affinity for $\mathrm{O}^{-}$of $2.7 \mathrm{eV}$. These may be compared with the experimental values of 0.754 and $1.462 \mathrm{eV}$, respectively. The $\mathrm{OH}$ bond energy obtained from a full CI calculation within this orbital space on that diatomic fragment is approximately $3.2 \mathrm{eV}$, considerably lower than the experimental value of $4.4 \mathrm{eV}$.

The data in Fig. 2 are the energies of CI roots from calculations on the states of the anion, and summed energies from calculations on the atom and diatom fragments. The energy of neutral $\mathrm{H}_{2} \mathrm{O}$, also from a full CI in the same basis, is included. Full CI is size-consistent and therefore this it is meaningful to compare these calculations.

\section{APPENDIX B: DETAILS OF SCATTERING CALCULATIONS}

The scattering calculations described in this paper were all performed using the complex Kohn variational method. This method makes use of a trial wave function that is expanded in terms of square-integrable (Cartesian Gaussian) and continuum basis functions that incorporate the correct asymptotic boundary conditions. Detailed descriptions of the method have been given elsewhere (see, for instance, refs. [48, 49]).

For a target with $N$ electrons, the trial wavefunction has the form

$$
\begin{aligned}
\Psi_{\Gamma_{0}}^{(+)}= & \sum_{\Gamma} \mathcal{A}\left[\chi_{\Gamma}\left(\mathbf{r}_{1} \ldots \mathbf{r}_{N}\right) F_{\Gamma, \Gamma_{0}}^{(+)}\left(\mathbf{r}_{N+1}\right)\right] \\
& +\sum_{\mu} d_{\mu}^{\Gamma_{0}} \Theta_{\mu}\left(\mathbf{r}_{1} \ldots \mathbf{r}_{N+1}\right) .
\end{aligned}
$$

The first sum in Eq. (B1) is over target states explicitly included in a close-coupling expansion, which may be energetically open or closed, and for which $\chi_{\Gamma}\left(\mathbf{r}_{1} \ldots \mathbf{r}_{N}\right)$ denotes the corresponding electronic state of the target molecule. The antisymmetrizer is denoted by $\mathcal{A}$, and the scattering orbital (channel eigenfunction) associated with channel $\Gamma$ is

$$
\begin{aligned}
F_{\Gamma, \Gamma_{0}}^{(+)}(\mathbf{r})= & \sum_{i} c_{i}^{\Gamma, \Gamma_{0}} \varphi_{i}(\mathbf{r}) \\
& +\sum_{l, m}\left[f_{l, m}\left(k_{\Gamma} r\right) \delta_{l, l_{0}} \delta_{m, m_{0}} \delta_{\Gamma, \Gamma_{0}}\right. \\
& \left.+T_{l, l_{0}, m, m_{0}}^{\Gamma, \Gamma_{0}} g_{l, m}^{(+)}\left(k_{\Gamma} r\right)\right] Y_{l, m}(\hat{\mathbf{r}}) / r
\end{aligned}
$$

for incoming boundary conditions in channel $\Gamma_{0}$. In Eq. (B2) $\varphi_{i}$ denotes a Gaussian molecu-

lar orbital, and $f_{l, m}\left(k_{\Gamma} r\right)$ and $g_{l, m}^{(+)}\left(k_{\Gamma} r\right)$ denote continuum functions which are regular at the 
origin and whose asymptotic forms correspond to Ricatti-Bessel and outgoing Ricatti-Hankel functions, respectively:

$$
\begin{array}{r}
f_{l, m}\left(k_{\Gamma} r\right) \underset{r \rightarrow \infty}{\longrightarrow} j_{l}\left(k_{\Gamma} r\right) / \sqrt{k_{\Gamma}} \\
g_{l, m}^{(+)}\left(k_{\Gamma} r\right) \underset{r \rightarrow \infty}{\longrightarrow} h_{l}^{(+)}\left(k_{\Gamma} r\right) / \sqrt{k_{\Gamma}} .
\end{array}
$$

With each of these continuum functions is associated a channel momentum, $k_{\Gamma}$ :

$$
k_{\Gamma}^{2} / 2=E-E_{\Gamma} .
$$

The second sum in Eq. (B1) is over square-integrable $(N+1)$-electron terms (configuration state functions) constructed from Gaussian molecular orbitals and incorporates correlation effects not described by the close-coupling expansion of the first sum. For convenience we refer to the $(N+1)$-electron configurations, $\Theta_{\mu}$, in the second sum as the "Q-space" and to the $(N+1)$-electron configurations explicitly involving target states as the "P-space" of the calculation.

For the calculations presented in Fig. 7, we used the same Gaussian basis as described in Appendix A. Using this primitive basis, we performed SCF calculations on ground state neutral water and on the ${ }^{2} B_{1}$ resonance state at the geometry $r_{1}=r_{2}=1.81, \theta=73^{\circ}$. For the neutral $\mathrm{H}_{2} \mathrm{O}$ calculation, we then obtained a correlating orbital of $B_{2}$ symmetry via a CI calculation including the SCF reference configuration as well as all double excitations from the $3 a_{1}$ and $1 b_{2}$ orbitals. The density matrix corresponding to the ground state CI root was diagonalized to obtain the correlating $b_{2}$ orbital.

For the ${ }^{2} B_{1}$ resonance calculation, we obtained $4 a_{1}, 5 a_{1}, 2 b_{2}$, and $2 b_{1}$ orbitals via a CI calculation in which the grandparent core configuration, $1 a_{1}^{2} 2 a_{1}^{2} 1 b_{2}^{2} 3 a_{1}^{2} 1 b_{1}^{1}$, was held constant, and a full CI was performed with the remaining two electrons in the remaining orbital space. Again, the density matrix of this CI calculation was diagonalized to produce natural orbitals.

The six orbitals from the neutral calculation - five SCF and one correlating - were combined with the four resonance orbitals from the resonance calculation. The resulting set was re-orthogonalized at each geometry. We found the calculation of the resonance trajectories shown in Fig. 7 to be extremely sensitive to variations in the basis set; this is why the orbitals were only calculated once.

Thirteen states $\chi_{\Gamma}$ were explicitly included in the P-space portion of the Kohn trial function in Eq. (B1). These states were defined as the roots of a complete active space CI 
within the space of the six neutral orbitals, with the $1 a_{1}$ and $2 a_{1}$ orbitals constrained to be doubly occupied and all single and double excitations into the other four resonance orbitals.

Similarly, the Q-space terms $\Theta_{\mu}$ were comprised of all configurations that could be generated by doubly occupying the $1 a_{1}$ and $2 a_{1}$ orbitals, and performing full CI with the remaining seven electrons in the space of the remaining four neutral orbitals, with single and double excitations into the space of the four resonance orbitals. This prescription for the Q-space avoids problems with recorrelation of the target states [49].

The expansion of the continuum functions $F_{\Gamma, \Gamma_{0}}^{(+)}$in Eq. (B2) included $Y_{l, m}$ 's spanning the range $l=0$ to 4 , with all symmetry-allowed values of $m$ included for each target state.

A careful fit of S-matrix elements was performed to obtain the real and imaginary parts of the energies of the nearly degenerate $1^{2} A^{\prime}$ and $2^{2} A^{\prime}$ resonances from complex Kohn calculations to produce the data plotted in Fig. 7 . At 30 energies near the resonance position, the values of two S-matrix elements, one $A_{1}$-like and the other $B_{1}$-like, were summed, and that sum was fit to a Breit-Wigner form with a background contribution given by a quartic polynomial in the energy. A fit using a quartic background was necessary to allow the two overlapping resonance profiles to be resolved.

For the full CI scattering calculations of subsections IV B and IV C, we used the smaller double zeta plus polarization and diffuse orbital set of Dunning [50, 51], but the same prescription for the orbital basis and target states as described for the full CI calculations in Appendix A, except we constrained the oxygen 1s orbital to be doubly occupied. The Q-space terms were described by full CI within the atomic orbital basis, with the $1 a_{1}$ orbital always doubly occupied. These calculations were 21-state close coupling calculations (21 target states included in the trial wavefunction, Eq. (B1)), and included $Y_{l, m}$ 's spanning $l=0$ to 5 .

\section{ACKNOWLEDGMENTS}

Work performed at the University of California Lawrence Berkeley National Laboratory was under the auspices of the US Department of Energy under Contract DE-AC0376SF00098 and was supported by the U.S. DOE Office of Basic Energy Sciences, Division 
of Chemical Sciences.

[1] D. J. Haxton, Z. Zhang, C. W. McCurdy, and T. N. Rescigno, Phys. Rev. A 69, 062713 (2003).

[2] D. J. Haxton, Z. Zhang, H.-D. Meyer, T. N. Rescigno, and C. W. McCurdy, Phys. Rev. A 69, 062714 (2003).

[3] W. N. Lozier, Phys. Rev. 36, 1417 (1930).

[4] I. S. Buchel'nikova, Zh. Eksperim. i Teor. Fiz. 35, 1119 (1959).

[5] G. J. Schultz, J. Chem. Phys. 44, 3856 (1966).

[6] R. N. Compton and L. G. Christophorou, Phys. Rev. 154, 110 (1967).

[7] C. E. Melton, J. Chem. Phys. 57, 4218 (1972).

[8] L. Sanche and G. J. Schultz, J. Chem. Phys. 58, 479 (1972).

[9] S. Trajmar and R. I. Hall, J. Phys. B. 7, L458 (1974).

[10] M. Jungen, J. Vogt, and V. Staemmler, Chem. Phys. 37, 49 (1979).

[11] D. S. Belic̀, M. Landau, and R. I. Hall, J. Phys. B. 14, 175 (1981).

[12] M. G. Curtis and I. C. Walker, J. Chem. Soc. Faraday Trans. 88, 2805 (1992).

[13] C. R. Claydon, G. A. Segal, and H. S. Taylor, J. Chem. Phys 54, 3799 (1971).

[14] S. Goursaud, M. Sizun, and F. Fiquet-Fayard, J. Chem. Phys 65, 5453 (1976).

[15] S. Goursaud, M. Sizun, and F. Fiquet-Fayard, J. Chem. Phys 68, 4310 (1978).

[16] T. J. Gil, T. N. Rescigno, C. W. McCurdy, and B. H. Lengsfield, III, Phys. Rev. A 49, 2642 (1994).

[17] M. A. D. Fluendy and I. C. Walker, J. Chem. Soc. Faraday Trans. 91, 2249 (1995).

[18] L. A. Morgan, J. Phys. B: At. Mol. Opt. Phys. 31, 5003 (1998).

[19] J. D. Gorfinkel, L. A. Morgan, and J. Tennyson, J. Phys. B 35, 543 (2002).

[20] D. T. Birtwistle and A. Herzenberg, J. Phys. B 4, 53 (1971).

[21] L. Dube and A. Herzenberg, Phys. Rev. A 20, 194 (1979).

[22] J. N. Bardsley and J. M. Wadehra, J. Chem. Phys. 78, 7227 (1983).

[23] T. F. O'Malley and H. S. Taylor, Phys. Rev. 176, 207 (1968).

[24] T. F. O'Malley, Phys. Rev. 150, 14 (1966).

[25] C. E. Klots and R. N. Compton, J. Chem. Phys. 69, 1644 (1978).

[26] J. L. Mauer and G. J. Schultz, Phys. Rev. A 7, 593 (1972). 
[27] F. C. Fehsenfeld, C. J. Howard, and E. E. Ferguson, J. Chem. Phys. 58, 5841 (1973).

[28] M. McFarland, D. L. Albritton, F. C. Fehsenfeld, E. E. Ferguson, and A. L. Schmeltekopf, J. Chem. Phys. 59, 6629 (1973).

[29] C. J. Howard, F. C. Fehsenfeld, and M. McFarland, J. Chem. Phys. 60, 5086 (1974).

[30] J. Comer and G. J. Schultz, J. Phys. B 7, L349 (1974).

[31] J. Comer and G. J. Schultz, Phys. Rev. A 10, 2100 (1974).

[32] R. van Harrevelt and M. C. van Hemert, J. Chem. Phys. 112, 5777 (2000).

[33] H. Estrada, L. S. Cederbaum, and W. Domcke, J. Chem. Phys. 84, 152 (1985).

[34] S. Feuerbacher, T. Sommerfeld, and L. S. Cederbaum, J. Chem. Phys. 120, 3201 (2004).

[35] S. Feuerbacher and L. S. Cederbaum, J. Chem. Phys. 121, 5 (2004).

[36] E. Balslev and J. M. Combes, Commun. Math. Phys. 22, 280 (1971).

[37] W. Reinhardt, Ann. Rev. Phys. Chem. 33, 223 (1982).

[38] J. D. Doll, T. F. George, and W. H. Miller, J. Chem. Phys. 58, 1343 (1973).

[39] Ovchinnikov, Phys. Rev. A 42, 3865 (1990).

[40] J. H. Macek and S. Y. Ovchinnikov, Phys. Rev. A 50, 468 (1994).

[41] G. Seng and F. Lindler, J. Phys. B 9, 2539 (1976).

[42] A. Jain and D. G. Thompson, J. Phys. B 16, L347 (1983).

[43] K. Rohr, J. Phys. B 10, L735 (1977).

[44] T. Nishimura and Y. Itikawa, J. Phys. B 28, 1995 (1995).

[45] A. A. A. El-Zein, M. J. Brunger, and W. R. Newell, J. Phys. B 33, 5033 (2000).

[46] O. Moreira, D. G. Thompson, and B. M. McLaughlin, J. Phys. B 34, 3737 (2001).

[47] M. Allan and O. Moreira, J. Phys. B: At. Mol. Phys. 35, L37 (2002).

[48] T. N. Rescigno, C. W. McCurdy, A. E. Orel, and B. H. Lengsfield III, in Computational Methods for Electron-Molecule Collisions, edited by W. M. Huo and F. A. Gianturco (Plenum, New York, 1995).

[49] T. N. Rescigno, B. H. Lengsfield III, and C. W. McCurdy, in Modern Electronic Structure Theory, edited by D. R. Yarkony (World Scientific, Singapore, 1995), vol. 1, pp. 501-588.

[50] J. T. H. Dunning, J. Chem. Phys. 53, 2823 (1970).

[51] J. T. H. Dunning and P. J. Hay, in Methods of Electronic Structure Theory, volume 2, edited by H. F. S. III (Plenum, New York, 1977). 